TRANSACTIONS OF THE

AMERICAN MATHEMATICAL SOCIETY

Volume 355, Number 3, Pages 901-924

S 0002-9947(02)03151-3

Article electronically published on October 1, 2002

\title{
HÖLDER REGULARITY FOR A KOLMOGOROV EQUATION
}

\author{
ANDREA PASCUCCI
}

\begin{abstract}
We study the interior regularity properties of the solutions to the degenerate parabolic equation,

$$
\Delta_{x} u+b \partial_{y} u-\partial_{t} u=f, \quad(x, y, t) \in \mathbb{R}^{N} \times \mathbb{R} \times \mathbb{R},
$$

which arises in mathematical finance and in the theory of diffusion processes.
\end{abstract}

\section{INTRODUCTION}

We consider the degenerate parabolic equation

$$
L_{b} u \equiv\left(\Delta_{x}+b \partial_{y}-\partial_{t}\right) u=f
$$

in the variables $(x, y, t) \in \mathbb{R}^{N} \times \mathbb{R} \times \mathbb{R}$, where $\Delta_{x}$ denotes the Laplacian operator acting in the variables $x=\left(x_{1}, \ldots, x_{N}\right)$. We aim to prove some new Schauder type interior estimates related to the Hölder classes $C_{b}^{k, \alpha}$ naturally associated to $L_{b}$. Our estimates improve the known ones and allow us to study nonlinear equations of the form

$$
\Delta_{x} u+b(\cdot, u) \partial_{y} u-\partial_{t} u=f(\cdot, u),
$$

recently considered in mathematical finance in [1] and [2. We also obtain regularity results for the following nonlinear convection-diffusion model proposed by Escobedo, Vazquez and Zuazua in [9]:

$$
\Delta_{x} u+\partial_{y} g(u)-\partial_{t} u=0
$$

with particular interest in the case $g(u)=u|u|^{q-1}$ for $\left.q \in\right] 1, \frac{N+2}{N+1}[$.

While we refer to the next section for the precise notation and assumptions on the coefficients $b$ and $f$, we would like to make some preliminary remarks. One of the main features of operator $L_{b}$ is the strong degeneracy of its characteristic form due to the lack of diffusion in the $y$-direction. On the other hand, $L_{b}$ can be represented in the form,

$$
\sum_{j=1}^{p} X_{j}^{2}+X_{p+1}
$$

where the first-order differential operators (vector fields) $X_{j}$ are defined as follows:

$$
X_{j}=\partial_{x_{j}}, j=1, \ldots, p=N, \quad \text { and } \quad X_{N+1}=b \partial_{y}-\partial_{t} .
$$

A classical result by Hörmander [11] states that if an operator $H$, in the form (1.3), is such that the vector fields $X_{j}$ have smooth coefficients and their commutators,

Received by the editors June 27, 2002.

2000 Mathematics Subject Classification. Primary 35K57, 35K65, 35K70.

Investigation supported by the University of Bologna. Funds for selected research topics. 
up to a certain order, span the whole space at every point, then $H$ is hypoelliptic. This means that every weak solution of $H u=f$, with $f \in C^{\infty}$, is smooth.

For instance, if $N=1$ and $b(x, y, t)=x$ in (1.1), then

$$
L_{x}=\partial_{x x}+x \partial_{y}-\partial_{t}
$$

is the linearized prototype of the Kolmogorov operator which, under suitable conditions, describes the probability density of a physical system with two degrees of freedom (cf. [18). In this case we have

$$
X_{1}=\partial_{x}, \quad X_{2}=x \partial_{y}-\partial_{t}, \quad \text { and } \quad\left[X_{1}, X_{2}\right] \equiv X_{1} X_{2}-X_{2} X_{1}=\partial_{y},
$$

so that $L_{x}$ is a hypoelliptic operator. More generally, the vector fields in (1.4) verify

$$
\left[X_{j}, X_{N+1}\right]=\left(\partial_{x_{j}} b\right) \partial_{y}, \quad j=1, \ldots, N \text {; }
$$

therefore, the assumptions of Hörmander's theorem are satisfied if

$$
b \in C^{\infty} \quad \text { and } \quad \nabla_{x} b \equiv\left(\partial_{x_{1}} b, \ldots, \partial_{x_{N}} b\right) \neq 0 .
$$

Hörmander's result was the starting point of an extensive study of operators $H$ in the form (1.3) with smooth vector fields. A general theory of the regularity analogous to the classical one has been developed both in Sobolev and Hölder spaces by Folland [10], Rothschild and Stein [17], Nagel and Stein [15], and Beals [3]. We also refer to the more recent papers by Krylov [12] and by Lanconelli, Polidoro and the author [13]. The case of operators in the form

$$
\sum_{i, j=1}^{p} a_{i j} X_{i} X_{j}+X_{p+1}
$$

with non-regular coefficients $a_{i j}$ has been considered by Xu [19] and Bramanti and Brandolini [4]. Thanks to the known results (cf. [17]), we have the following.

Theorem 1.1 (Rothschild-Stein). Let $u$ be a classical solution of 1.1 (cf. Definition 4.1) in an open subset $\Omega$ of $\mathbb{R}^{N+2}$. If $f \in C_{b}^{k-2, \alpha}(\Omega)$ and the Hörmander condition (1.7) holds, then $u \in C_{b}^{k, \alpha}(\Omega)$.

The regularity assumption on $b$ in Theorem 1.1 can be weakened by assuming at least $b \in C_{b}^{k+1, \alpha}(\Omega)$. In this case the proof follows the original one with minor changes and we obtain the following:

Theorem 1.2 (Rothschild-Stein). Let $u$ be a classical solution of 1.1 in $\Omega$ with $f \in C_{b}^{k-2, \alpha}(\Omega)$. If $b \in C_{b}^{k+1, \alpha}(\Omega)$ and $\nabla_{x} b \neq 0$ in $\Omega$, then $u \in C_{b}^{k, \alpha}(\Omega)$.

In view of the classical Schauder estimates, the previous results do not seem optimal. In particular, we emphasize that they do not allow the treatment of the existence and regularity theory of nonlinear equations. As a matter of fact, the further weaker assumption $b \in C_{b}^{k-2, \alpha}$ is naturally expected. Actually, the techniques used by Rothschild and Stein require the smoothness of the vector fields as an essential hypothesis. On the contrary, here we aim to consider non-regular vector fields.

In the recent papers [7], 8] in collaboration with Citti and Polidoro, we considered the nonlinear equation in three variables

$$
L_{u} u=\partial_{x x} u+u \partial_{y} u-\partial_{t} u=f
$$


and we studied the regularity of the solution $u$ by a modification of the classical freezing method. More precisely, we regarded $L_{b}$ as a local perturbation of a Hörmander's operator on the Heisenberg group. This last operator played the same role as the constant coefficients operators in the classical theory. This technique was introduced by Citti in [5] to study an equation of Levi type.

Aiming to adapt those ideas, we immediately realize that, in dimensions higher than three, the Lie algebra formally associated to $L_{b}$ is not free. This means that the vector fields $X_{j}$ do not satisfy as few linear relations as possible (i.e., only those forced by anti-commutativity and the Jacobi identity). As a consequence, the algebra that one might naturally associate to $L_{b}$ varies from point to point. In order to overcome this problem and to eliminate the inessential relations among the commutators, we add some extra variables and we lift the operator $L_{b}$ to a higher-dimensional space. We recall that a general version of the so-called "lifting method" for an operator in (1.3) with smooth coefficients, is due to Rothschild and Stein [17. In our case we make the tentative choice to define the following operator in $\mathbb{R}^{2 N+1}$ :

$$
L_{B}=\Delta_{x}+b \partial_{y_{1}}+x_{2} \partial_{y_{2}}+\ldots+x_{N} \partial_{y_{N}}-\partial_{t}
$$

where $(x, y, t)=\left(x_{1}, \ldots, x_{N}, y_{1}, \ldots, y_{N}, t\right)$ denotes the point in $\mathbb{R}^{2 N+1}$. In order to apply to (1.9) the freezing techniques cited above, a detailed analysis and careful estimates of the fundamental solutions to the frozen operators are in order. This is done in Section 3 and it is our main proof. Then, we study the regularity properties of $L_{B}$ and finally, we apply our results to the operator $L_{b}$. We prove the following.

Theorem 1.3. Let $u$ be a classical solution of (1.1) in $\Omega$ (cf. Definition 4.1 and Remark 4.3) and assume the Hörmander type condition

$$
b \in C^{1}(\Omega) \quad \text { and } \quad \nabla_{x} b \neq 0 \quad \text { in } \Omega .
$$

If $b, f \in C_{b}^{k-2, \alpha}(\Omega)$, with $k \geq 2$ and $\left.\alpha \in\right] 0,1\left[\right.$, then $u \in C_{b}^{k, \bar{\alpha}}(\Omega)$ for every $\left.\bar{\alpha} \in\right] 0, \alpha[$.

We remark that Theorem 1.3 and a bootstrap argument give simple conditions for the interior regularity of solutions to a nonlinear equation of the form (1.2). In particular, we refine the results in [7].

Two possible directions for extending Theorem 1.3 come readily to mind. It seems that our technique can be adapted without difficulty to the following, more general, class of ultraparabolic operators in $\mathbb{R}^{N+2}$ :

$$
\sum_{i, j=1}^{N} a_{i j} \partial_{x_{i} x_{j}}+b \partial_{y}-\partial_{t}
$$

where $\left(a_{i j}\right)$ is a positive definite matrix with Hölder continuous entries. Secondly, assumption (1.10) could be relaxed by a "higher step" condition, that is, by requiring that higher-order commutators of the vector fields $X_{j}$ span $\mathbb{R}^{N+2}$. In this case, it seems that the proof would be essentially analogous, even if it could become considerably knotty.

This paper is organized as follows. In Section 2 we set the notation and we collect some tools for the analysis on nilpotent Lie groups. In Section 3 we provide some estimates of the fundamental solutions of the frozen operators. Section 4 is devoted to the proof of Theorem 1.3 . 


\section{HÖLDER CLASSES AND CONTROL DISTANCES}

In this section we present some preliminary material and we define the lifted and frozen operators related to $L_{b}$. We begin by defining the Hölder classes related to the vector fields in (1.4). For the reader's convenience, we also give the following standard

Definition 2.1. Let $D$ be a locally Lipschitz continuous vector field on $\Omega$ and $d$ a (positive) formal degree associated to $D$. We call $u$ Hölder continuous with exponent $\alpha, \alpha \in] 0, d\left[\right.$, in $\Omega$ w.r.t. $D$ and we write $u \in C_{D}^{\alpha}(\Omega)$ if, for every compact subset $E$ of $\Omega$, there exists a constant $C$ such that

$$
|u(\exp (\delta D)(z))-u(z)| \leq C|\delta|^{\frac{\alpha}{d}}
$$

for every $z \in E$ and suitably small $\delta$. We refer, for instance, to [16] for the definition and properties of exponential mappings induced by vector fields. We say that $u$ is Lie derivable w.r.t. $D$ in $z \in \Omega$ if the following limit exists:

$$
D u(z)=\lim _{\delta \rightarrow 0} \frac{u(\exp (\delta D)(z))-u(z)}{\delta} .
$$

Definition 2.2. Let $\alpha \in] 0,1\left[\right.$. We set the formal degree of $X_{1}, \ldots, X_{N}$ in (1.4) equal to one and the formal degree of $X_{N+1}$ equal to two. We define

$$
C_{b}^{\alpha}(\Omega)=\bigcap_{j=1}^{N+1} C_{X_{j}}^{\alpha}(\Omega) .
$$

We say that $u \in C_{b}^{1, \alpha}(\Omega)$ if

$$
X_{j} u \in C_{b}^{\alpha}(\Omega), j=1, \ldots, N, \quad \text { and } \quad u \in C_{X_{N+1}}^{1+\alpha}(\Omega) .
$$

Finally, if $k \in \mathbb{N}, k \geq 2$, we define by recurrence the class $C_{b}^{k, \alpha}(\Omega)$ as follows: assuming that $b \in C_{b}^{k-2, \alpha}(\Omega)$, we say that $u \in C_{b}^{k, \alpha}(\Omega)$ if

$$
X_{j} u \in C_{b}^{k-1, \alpha}(\Omega), j=1, \ldots, N, \quad \text { and } \quad X_{N+1} u \in C_{b}^{k-2, \alpha}(\Omega) .
$$

For greater convenience, when in the sequel we consider the class $u \in C_{b}^{k, \alpha}(\Omega)$ with $k \geq 2$, we always implicitly assume that $b \in C_{b}^{k-2, \alpha}(\Omega)$.

No regularity in the $y$-direction is seemingly assumed in the definition of $C_{b}^{k, \alpha}(\Omega)$. On the other hand, keeping in mind that $\left[X_{j}, X_{N+1}\right]=\left(\partial_{x_{j}} b\right) \partial_{y}$ and Hörmander's condition, it is natural to set the formal degree of the vector field $\partial_{y}$ equal to three and it is possible to prove, by a standard argument based on the CampbellHausdorff formula, the following

Lemma 2.3. If $k=0,1,2$ and $u \in C_{b}^{k, \alpha}(\Omega)$, then $u \in C_{\partial_{y}}^{k+\alpha}(\Omega)$. If $k \geq 3$ and $u \in C_{b}^{k, \alpha}(\Omega)$, then $\partial_{y} u \in C_{b}^{k-3, \alpha}(\Omega)$.

By the previous lemma, we have the following inclusion of the space $C_{b}^{k, \alpha}$ in the space of Hölder continuous functions in the classical sense:

$$
C_{b}^{3 k, \alpha}(\Omega) \subseteq C^{k, \frac{\alpha}{3}}(\Omega)
$$

We now lift the original vector fields in (1.4) to $\mathbb{R}^{2 N+1}$ in such a way that they become free. Since we aim to prove a local result, it is not restrictive to suppose that $\Omega$ is suitably small. Then, without loss of generality, by (1.10), we may assume that 
$\partial_{x_{1}} b \neq 0$ in $\Omega$. In the sequel we denote by $z=(x, y, t)=\left(x_{1}, \ldots, x_{N}, y_{1}, \ldots, y_{N}, t\right)$ and $\zeta=(\xi, \eta, \tau)$ the points in $\mathbb{R}^{2 N+1}$ and we set $\Omega_{0}=\Omega \times \mathbb{R}^{N-1}$.

We define the lifted vector fields on $\Omega_{0}$ as

$$
D_{j}=\partial_{x_{j}}, j=1, \ldots, N, \quad D_{N+1}=\left\langle B, \nabla_{y}\right\rangle-\partial_{t},
$$

where

$$
B\left(x, y_{1}, t\right)=\left(b\left(x, y_{1}, t\right), x_{2}, \ldots, x_{n}\right), \quad \text { and } \quad \nabla_{y}=\left(\partial_{y_{1}}, \ldots, \partial_{y_{N}}\right) .
$$

Thus, the operator $L_{B}$ in (1.9) can be expressed in the form

$$
L_{B}=\sum_{j=1}^{N} D_{j}^{2}+D_{N+1} .
$$

Since $\partial_{x_{1}} b \neq 0$ in $\Omega_{0}$, the commutators

$$
\begin{gathered}
D_{N+2} \equiv\left[D_{1}, D_{N+1}\right]=\left(\partial_{x_{1}} b\right) \partial_{y_{1}}, \\
D_{N+1+j} \equiv\left[D_{j}, D_{N+1}\right]=\left(\partial_{x_{j}} b\right) \partial_{y_{1}}+\partial_{y_{j}}, \quad 2 \leq j \leq N,
\end{gathered}
$$

are linearly independent and the system $\left(D_{j}\right)_{1 \leq j \leq 2 N+1}$ forms a basis of $\mathbb{R}^{2 N+1}$ at every point of $\Omega_{0}$. Analogously to Definition 2.2 we give the notion of Hölder continuity related to $\left(D_{j}\right)$.

Definition 2.4. Let $\alpha \in] 0,1\left[\right.$. We set the formal degree of $D_{1}, \ldots, D_{N}$ equal to one and the formal degree of $D_{N+1}$ equal to two. We define

$$
C_{B}^{\alpha}\left(\Omega_{0}\right)=\bigcap_{j=1}^{N+1} C_{D_{j}}^{\alpha}\left(\Omega_{0}\right)
$$

We say that $u \in C_{B}^{1, \alpha}\left(\Omega_{0}\right)$ if

$$
D_{j} u \in C_{B}^{\alpha}\left(\Omega_{0}\right), j=1, \ldots, N, \quad \text { and } \quad u \in C_{D_{N+1}}^{1+\alpha}\left(\Omega_{0}\right) .
$$

Finally, if $k \in \mathbb{N}, k \geq 2$, we define by recurrence the class $C_{B}^{k, \alpha}\left(\Omega_{0}\right)$ as follows: assuming that $b \in C_{b}^{k-2, \alpha}(\Omega)$, we say that $u \in C_{B}^{k, \alpha}\left(\Omega_{0}\right)$ if

$$
D_{j} u \in C_{B}^{k-1, \alpha}\left(\Omega_{0}\right), j=1, \ldots, N, \quad \text { and } \quad D_{N+1} u \in C_{B}^{k-2, \alpha}\left(\Omega_{0}\right) .
$$

Remark 2.5. Given a function $w=w\left(x, y_{1}, t\right)$ on $\Omega$, we denote again by $w$ its extension to $\Omega_{0}=\Omega \times \mathbb{R}^{N-1}$, i.e., the function defined by $w\left(x, y_{1}, \ldots, y_{N}, t\right)=$ $w\left(x, y_{1}, t\right)$. Hence, it is clear that a solution $u$ to (1.1) in $\Omega$ is also a solution to $L_{B} u=f$ in $\Omega_{0}$. Moreover, $u \in C_{b}^{k, \alpha}(\Omega)$ if and only if $u \in C_{B}^{k, \alpha}\left(\Omega_{0}\right)$.

We next construct a nilpotent Hörmander operator locally approximating $L_{B}$ and we introduce some distances naturally associated to the vector fields $D_{j}$ in (2.1). More details about distances defined by vector fields can be found in [10] and 16 .

For fixed $\bar{z} \in \Omega_{0}$, we define the frozen vector fields

$$
D_{j}^{\bar{z}}=\partial_{x_{j}}, \quad j=1, \ldots, N,
$$

and

$$
D_{N+1}^{\bar{z}}=\left(b(\bar{z})+\left\langle\nabla_{x} b(\bar{z}),(x-\bar{x})\right\rangle\right) \partial_{y_{1}}+\sum_{j=2}^{N} x_{j} \partial_{y_{j}}-\partial_{t} .
$$


Since the commutators of $D_{j}^{\bar{z}}$ and $D_{N+1}^{\bar{z}}$ are given by

$$
\begin{aligned}
D_{N+2}^{\bar{z}} & \equiv\left[D_{1}^{\bar{z}}, D_{N+1}^{\bar{z}}\right]=\partial_{x_{1}} b(\bar{z}) \partial_{y_{1}}, \\
D_{N+1+j}^{\bar{z}} & \equiv\left[D_{j}^{\bar{z}}, D_{N+1}^{\bar{z}}\right]=\partial_{x_{j}} b(\bar{z}) \partial_{y_{1}}+\partial_{y_{j}}, \quad 2 \leq j \leq N,
\end{aligned}
$$

and $\partial_{x_{1}} b(\bar{z}) \neq 0$ by assumption, the Hörmander condition is verified and the operator

$$
L_{\bar{z}}=\sum_{j=1}^{N}\left(D_{j}^{\bar{z}}\right)^{2}+D_{N+1}^{\bar{z}}
$$

is hypoelliptic. We call

$$
\nabla_{\bar{z}} \equiv\left(D_{1}^{\bar{z}}, \ldots, D_{2 N+1}^{\bar{z}}\right),
$$

the intrinsic gradient related to the system of vector fields defined in (2.2) and (2.3).

For fixed $z \in \mathbb{R}^{2 N+1}$, we consider the exponential map

$$
E_{z}^{\bar{z}}(\theta)=\exp \left(\left\langle\theta, \nabla_{\bar{z}}\right\rangle\right)(z), \quad \theta \in \mathbb{R}^{2 N+1} .
$$

It is well known that the map $E_{z}^{\bar{z}}$ is a global diffeomorphism. Its inverse function $\theta_{z}^{\bar{z}}$ is usually called the canonical change of coordinates and it has the explicit expression

$$
\theta_{z}^{\bar{z}}(\zeta)=\left(E_{z}^{\bar{z}}\right)^{-1}(\zeta)=\left(\theta_{1}, \ldots, \theta_{2 N+1}\right),
$$

where

$$
\begin{aligned}
\theta_{j} & =\xi_{j}-x_{j}, \quad 1 \leq j \leq N, \\
\theta_{N+1} & =-(\tau-t), \\
\theta_{N+2}= & \frac{1}{\partial_{x_{1}} b(\bar{z})}\left[\eta_{1}-y_{1}+(\tau-t)\left(b(\bar{z})+\frac{\partial_{x_{1}} b(\bar{z})}{2}\left(\xi_{1}+x_{1}-2 \bar{x}_{1}\right)\right)\right. \\
& \left.\quad-\sum_{j=2}^{N} \partial_{x_{j}} b(\bar{z})\left(\eta_{j}-y_{j}+\bar{x}_{j}(\tau-t)\right)\right], \\
\theta_{N+1+j}= & \eta_{j}-y_{j}+\frac{(\tau-t)\left(\xi_{j}+x_{j}\right)}{2}, \quad 2 \leq j \leq N .
\end{aligned}
$$

Through the canonical change of coordinates, the vector fields $D_{j}^{z}, D_{j}^{\bar{z}}$ corresponding to different points $z, \bar{z} \in \Omega_{0}$, coincide. More precisely, if we set

$$
\begin{aligned}
D_{j}^{H} & =\partial_{\theta_{j}}-\frac{\theta_{N+1}}{2} \partial_{\theta_{N+1+j}}, \quad 1 \leq j \leq N, \\
D_{N+1}^{H} & =\partial_{\theta_{N+1}}+\frac{1}{2} \sum_{j=1}^{N} \theta_{j} \partial_{\theta_{N+1+j}}, \\
D_{N+1+j}^{H} & \equiv\left[D_{j}^{H}, D_{N+1}^{H}\right]=\partial_{\theta_{N+1+j}}, \quad 1 \leq j \leq N,
\end{aligned}
$$

then, for any smooth function $\varphi$ and $\bar{z} \in \Omega_{0}$, it follows that

$$
D_{j}^{\bar{z}}\left(\varphi \circ \theta_{\bar{z}}^{\bar{z}}\right)=\left(D_{j}^{H} \varphi\right) \circ \theta_{\bar{z}}^{\bar{z}}, \quad 1 \leq j \leq 2 N+1 .
$$

The vector fields in (2.7) generate a free Lie algebra which is isomorphic to the Heisenberg one. Indeed, the vector fields in (2.7) induce a composition law in $\mathbb{R}^{2 N+1}$ formally defined by the Campbell-Hausdorff formula, or explicitly

$$
(\theta \oplus \bar{\theta})_{j}= \begin{cases}\theta_{j}+\bar{\theta}_{j}, & \text { if } 1 \leq j \leq N+1, \\ \theta_{j}+\bar{\theta}_{j}+\frac{1}{2}\left(\theta_{j-N-1} \bar{\theta}_{N+1}-\bar{\theta}_{j-N-1} \theta_{N+1}\right), & \text { if } N+2 \leq j \leq 2 N+1,\end{cases}
$$


and the dilations group

$$
\delta_{\lambda}(\theta)=\left(\lambda \theta_{1}, \ldots, \lambda \theta_{N}, \lambda^{2} \theta_{N+1}, \lambda^{3} \theta_{N+2}, \ldots, \lambda^{3} \theta_{2 N+1}\right), \quad \lambda>0 .
$$

The space $\mathbb{R}^{2 N+1}$ endowed with the law $\oplus$ and the dilations $\delta_{\lambda}$ is a homogeneous Lie group. The associated Lie algebra of the $\oplus$-left-invariant vector fields is the one generated by $D_{1}^{H}, \ldots, D_{N+1}^{H}$. We also remark that $D_{1}^{H}, \ldots, D_{N}^{H}$ are $\delta_{\lambda}$-homogeneous of degree one and $D_{N+1}^{H}$ is $\delta_{\lambda}$-homogeneous of degree two. Therefore, the Hörmander operator,

$$
L_{H}=\sum_{j=1}^{N}\left(D_{j}^{H}\right)^{2}+D_{N+1}^{H},
$$

has a fundamental solution $\Gamma_{H}$ which is invariant with respect to the left $\oplus$ translations and it is homogeneous of degree $-Q+2$ where $Q=4 N+2$ is the homogeneous dimension of $\left(\mathbb{R}^{N+1}, \oplus\right)$. An explicit expression of $\Gamma_{H}$ is known (see, e.g., [14]); however, here we only use its qualitative properties. A norm homogeneous w.r.t. the dilations in $(2.8)$ is given by

$$
\|\theta\|_{H}=\sum_{j=1}^{N}\left(\left|\theta_{j}\right|+\left|\theta_{N+1+j}\right|^{\frac{1}{3}}\right)+\left|\theta_{N+1}\right|^{\frac{1}{2}},
$$

and the associated control distance is defined by

$$
d_{H}(\bar{\theta}, \theta)=\left\|\theta^{-1} \oplus \bar{\theta}\right\|_{H} .
$$

The following Lie product on $\mathbb{R}^{2 N+1}$ is naturally induced:

$$
\begin{aligned}
z \circ \zeta & =E_{\bar{z}}^{\bar{z}}\left(\theta_{\bar{z}}^{\bar{z}}(z) \oplus \theta_{\bar{z}}^{\bar{z}}(\zeta)\right) \\
& =\left(x+\xi-\bar{x}, y+\eta-\bar{y}-(\tau-\bar{t}) \mathcal{J}_{x} B(\bar{z})(x-\bar{x}), t+\tau-\bar{t}\right),
\end{aligned}
$$

where $\mathcal{J}_{x} B$ denotes the Jacobian matrix of $B$ w.r.t. the variable $x$, i.e., the diagonal matrix $\operatorname{diag}\left(\partial_{x_{1}} b, 1, \ldots, 1\right)$. Correspondingly, we have the dilations group

$$
\delta_{\lambda}^{\bar{z}}(z)=E_{\bar{z}}^{\bar{z}}\left(\delta_{\lambda} \theta_{\bar{z}}^{\bar{z}}(z)\right), \quad \lambda>0,
$$

and the associated control distance

$$
d_{\bar{z}}(z, \zeta)=\left\|\theta_{z}^{\bar{z}}(\zeta)\right\|_{H} .
$$

Lemma 2.6. There exists a constant $c_{0}$, only dependent on $\Omega_{0}$, such that

$$
c_{0}^{-1} d_{z}(z, \zeta) \leq \tilde{d}_{z}(z, \zeta) \leq c_{0} d_{z}(z, \zeta),
$$

for every $z, \zeta \in \Omega_{0}$, where

$$
\begin{aligned}
\tilde{d}_{z}(z, \zeta)= & |x-\xi|+|t-\tau|^{\frac{1}{2}}+\left|y_{1}-\eta_{1}+(t-\tau) b(z)\right|^{\frac{1}{3}} \\
& +\sum_{j=2}^{N}\left|y_{j}-\eta_{j}+\frac{(t-\tau)\left(\xi_{j}+x_{j}\right)}{2}\right|^{\frac{1}{3}}
\end{aligned}
$$


Proof. By 2.6) and by denoting $\theta_{z}^{z}(\zeta)=\left(\theta_{1}, \ldots, \theta_{2 N+1}\right)$, we have

$$
\begin{aligned}
\theta_{N+2}=\frac{1}{\partial_{x_{1}} b(z)}[ & \eta_{1}+y_{1}+b(z)(\tau-t)-\sum_{j=2}^{N} \partial_{x_{j}} b(z)\left(\eta_{j}-y_{j}+\frac{(\tau-t)\left(\xi_{j}+x_{j}\right)}{2}\right) \\
& \left.+\frac{1}{2} \sum_{j=2}^{N} \partial_{x_{j}} b(z)(\tau-t)\left(\xi_{j}-x_{j}\right)\right]+\frac{(\tau-t)\left(\xi_{1}-x_{1}\right)}{2} \\
= & \frac{1}{\partial_{x_{1}} b(z)}\left[\eta_{1}+y_{1}+b(z)(\tau-t)-\sum_{j=2}^{N} \partial_{x_{j}} b(z)\left(\theta_{N+1+j}+\frac{1}{2} \theta_{j} \theta_{N+1}\right)\right] \\
& -\frac{1}{2} \theta_{1} \theta_{N+1} .
\end{aligned}
$$

Hence (2.13) is an immediate consequence of the elementary inequality

$$
(a b)^{\frac{1}{3}} \leq \frac{a}{3}+\frac{2 \sqrt{b}}{3}, \quad \forall a, b>0 .
$$

We stress that the distances $d_{z}, d_{\zeta}$ corresponding to different points $z, \zeta \in \Omega_{0}$ are not, in general, equivalent. Nevertheless, using Lemma 2.6 it is straightforward to prove the following

Lemma 2.7. There exists a constant $c_{0}$, only dependent on $\Omega_{0}$, such that

$$
c_{0}^{-1} d_{z}(z, \bar{z}) \leq d_{\bar{z}}(\bar{z}, z) \leq c_{0} d_{z}(z, \bar{z})
$$

and

$$
d_{z}(z, \zeta) \leq c_{0}\left(d_{z}(z, \bar{z})+d_{\bar{z}}(\bar{z}, \zeta)\right)
$$

for every $z, \bar{z}, \zeta \in \Omega_{0}$.

It is remarkable that the Hölder continuity property related to the vector fields $D_{j}$ can be expressed in terms of the control distances associated to the frozen vector fields. Indeed, we have

Lemma 2.8. Let $g$ be a function on $\Omega_{0}$ and $\left.\alpha \in\right] 0,1[$. Suppose that, for every compact subset $E$ of $\Omega_{0}$, there exists a constant $C$ such that

$$
|g(z)-g(\bar{z})| \leq C d_{\bar{z}}(\bar{z}, z)^{\alpha}, \quad \forall z, \bar{z} \in E .
$$

Then $g \in C_{B}^{\alpha}\left(\Omega_{0}\right)$.

Proof. We have to prove that

$$
\begin{aligned}
\left|u\left(\exp \left(\delta D_{j}\right)(z)\right)-u(z)\right| & \leq c \delta^{\alpha}, \quad 1 \leq j \leq N, \\
\left|u\left(\exp \left(\delta D_{N+1}\right)(z)\right)-u(z)\right| & \leq c \delta^{\frac{\alpha}{2}} .
\end{aligned}
$$

The first inequality is obvious since $d_{z}\left(z, \exp \left(\delta D_{j}\right)(z)\right)=\delta$, for $1 \leq j \leq N$. With regard to the second inequality in (2.18), by assumption (2.17), it suffices to verify that

$$
d_{z}\left(z, \exp \left(\delta D_{N+1}\right)(z)\right) \leq c \delta^{\frac{1}{2}} .
$$


Denoting $\gamma(\delta)=\exp \left(\delta D_{N+1}\right)(z)$, we have

$$
\begin{aligned}
\gamma(\delta) & =z+\int_{0}^{\delta} D_{N+1}(\gamma(s)) d s \\
& =\left(x, y_{1}+\int_{0}^{\delta} b(\gamma(s)) d s, y_{2}+\delta x_{2}, \ldots, y_{N}+\delta x_{N}, t-\delta\right) .
\end{aligned}
$$

Hence, by Lemma 2.6. we get

$$
\begin{aligned}
c_{0}^{-1} d_{z}(z, \gamma(\delta)) \leq \tilde{d}_{z}(z, \gamma(\delta)) & =|\delta|^{\frac{1}{2}}+\left|\int_{0}^{\delta}(b(\gamma(s))-b(z)) d s\right|^{\frac{1}{3}} \\
& \leq|\delta|^{\frac{1}{2}}+\left|\delta \sup _{s \in[0, \delta]}\right| b(\gamma(s))-b(z)||^{\frac{1}{3}}
\end{aligned}
$$

(since $b \in C^{1}(\Omega)$ and $|z-\zeta| \leq d_{z}(z, \zeta)$ for every $z, \zeta$ in a suitably compact subset $E_{0}$ of $\Omega$ )

$$
\leq|\delta|^{\frac{1}{2}}+\left|\delta\left(\sup _{E_{0}}|\nabla b|\right) d_{z}(z, \gamma(\delta))\right|^{\frac{1}{3}}
$$

(by (2.14) for $\varepsilon>0$ )

$$
\leq|\delta|^{\frac{1}{2}}\left(1+\frac{2}{3}\left(\sup _{E_{0}}|\nabla b|\right)^{\frac{1}{2}}\right)+\frac{d_{z}(z, \gamma(\delta))}{3 \varepsilon},
$$

which yields (2.19) if $\varepsilon$ is suitably large.

The control distances previously introduced also give an estimate of the error in the intrinsic Taylor expansion of a function $u \in C_{B}^{k, \alpha}$. To be more precise, as in [7], Theorem 2.16, the following result can be proved.

Proposition 2.9. Let $\bar{z} \in \Omega_{0}$ and $u \in C_{B}^{k, \alpha}\left(\Omega_{0}\right)$. There exists a unique polynomial function $P_{\bar{z}}^{k} u$ which is a sum of terms $\delta_{\bar{\lambda}}^{\bar{z}}$-homogeneous of degree less than or equal to $k$ and verifies

$$
u(z)=P_{\bar{z}}^{k} u(z)+O\left(d_{\bar{z}}(\bar{z}, z)^{k+\alpha}\right), \quad \text { as } z \longrightarrow \bar{z} .
$$

For instance, in the case $k=0,1$, we have

$$
P_{\bar{z}}^{0} u(z)=u(\bar{z}) \quad \text { and } \quad P_{\bar{z}}^{1} u(z)=u(\bar{z})+\left\langle\nabla_{x} u(\bar{z}), x-\bar{x}\right\rangle .
$$

Hence, the frozen vector fields defined in (2.2) are obtained by considering the firstorder (intrinsic) Taylor expansion of the coefficients of the original vector fields in (2.1). In particular, we have

$$
D_{N+1}-D_{N+1}^{\bar{z}}=\left(b-P_{\bar{z}}^{1} b\right) \partial_{y_{1}} .
$$

We end this section by stating a technical lemma, which will be used in the proof of Theorem 1.3. For fixed $z, \bar{z} \in \Omega_{0}$ and a constant $M \geq 1$, we define the set

$$
\Omega_{M}(\bar{z}, z)=\left\{\zeta \in \Omega_{0} \mid M d_{\bar{z}}(\bar{z}, z) \leq d_{\bar{z}}(\bar{z}, \zeta)\right\} .
$$


We remark that we can choose $M$ sufficiently large so that

$$
\left(c_{M}\right)^{-1} d_{z}(z, \zeta) \leq d_{\bar{z}}(\bar{z}, \zeta) \leq c_{M} d_{z}(z, \zeta), \quad \forall \zeta \in \Omega_{M}(\bar{z}, z)
$$

for some constant $c_{M}$. Indeed, by Lemma 2.7 we have

$d_{z}(z, \zeta) \leq c_{0}\left(d_{z}(z, \bar{z})+d_{\bar{z}}(\bar{z}, \zeta)\right) \leq c_{0}\left(c_{0} d_{\bar{z}}(\bar{z}, z)+d_{\bar{z}}(\bar{z}, \zeta)\right) \leq c_{0}\left(\frac{c_{0}}{M}+1\right) d_{\bar{z}}(\bar{z}, \zeta)$, and, on the other hand,

$$
d_{\bar{z}}(\bar{z}, \zeta) \leq c_{0}\left(d_{\bar{z}}(\bar{z}, z)+d_{z}(z, \zeta)\right) \leq c_{0}\left(\frac{d_{\bar{z}}(\bar{z}, \zeta)}{M}+d_{z}(z, \zeta)\right),
$$

so that

$$
\left(\frac{1}{c_{0}}-\frac{1}{M}\right) d_{\bar{z}}(\bar{z}, \zeta) \leq d_{z}(z, \zeta)
$$

Lemma 2.10. Let $u \in C_{B}^{k, \alpha}\left(\Omega_{0}\right)$ and $k \leq 4$. For every compact subset $E$ of $\Omega_{0}$, there exists a constant $c=c(E)$ such that

$$
\begin{aligned}
\left|P_{z}^{k} u(\zeta)-P_{\bar{z}}^{k} u(\zeta)\right| & \leq c d_{\bar{z}}(\bar{z}, z)^{\alpha} d_{\bar{z}}(\bar{z}, \zeta)^{k}, \\
\left|\left(P_{z}^{k} u(\zeta)-P_{z}^{1} u(\zeta)\right)-\left(P_{\bar{z}}^{k} u(\zeta)-P_{\bar{z}}^{1} u(\zeta)\right)\right| & \leq c d_{\bar{z}}(\bar{z}, z)^{\alpha} d_{\bar{z}}(\bar{z}, \zeta)^{k},
\end{aligned}
$$

for $z, \bar{z} \in E$ and $\zeta \in \Omega_{M}(\bar{z}, z)$.

Proof. The proof is a direct and tiresome computation. We only show (2.23) for $k=1$. We have

$$
\left|P_{z}^{1} u(\zeta)-P_{\bar{z}}^{1} u(\zeta)\right|=\left|u(z)-P_{\bar{z}}^{1} u(z)+\left\langle\xi-x, \nabla_{x}(u(z)-u(\bar{z}))\right\rangle\right|
$$

(since $u \in C_{B}^{1, \alpha}\left(\Omega_{0}\right)$ and by (2.22) $)$

$$
\leq c d_{\bar{z}}(\bar{z}, z)^{\alpha} d_{\bar{z}}(\bar{z}, \zeta) .
$$

\section{PARAmetrices}

The proof of Theorem 1.3 is based on a representation formula for classical solutions to (1.1) in terms of the fundamental solution $\Gamma_{\bar{z}}$ of the frozen operator $L_{\bar{z}}$ in (2.4). In this section, we provide some crucial estimates of $\Gamma_{\bar{z}}$, with $\bar{z} \in$ $\Omega_{0}=\Omega \times \mathbb{R}^{N-1}$ (cf. Proposition 3.1). Most of the results of this section are rather technical.

We denote by $\Gamma_{\bar{z}}(z, \zeta)$ (resp. $\left.\Gamma_{H}(\theta)\right)$ the fundamental solution of $L_{\bar{z}}$ (resp. of $L_{H}$ in (2.9) ), evaluated in $z$ (resp. in $\theta$ ) and with pole in $\zeta$ (resp. in the origin). We note that

$$
\Gamma_{\bar{z}}(z, \zeta)=\Gamma_{\bar{z}}\left(\zeta^{-1} \circ z, 0\right)=\frac{1}{\partial_{x_{1}} b(\bar{z})} \Gamma_{H}\left(\theta_{\bar{z}}^{\bar{z}}\left(\zeta^{-1} \circ z\right)\right),
$$

where the product "o" is defined in (2.11).

We introduce some auxiliary notation. We denote the identity by $D_{0}=D_{0}^{\bar{z}}=$ $D_{0}^{H}$ and for every multi-index $\sigma=\left(\sigma_{1}, \ldots, \sigma_{m}\right) \in\{0,1, \ldots, 2 N+1\}^{m}$, we set

$$
D_{\sigma}^{\bar{z}}=D_{\sigma_{1}}^{\bar{z}} \cdots D_{\sigma_{m}}^{\bar{z}}, \quad D_{\sigma}^{H}=D_{\sigma_{1}}^{H} \cdots D_{\sigma_{m}}^{H} .
$$

We call the weight of $\sigma$ the number

$$
|\sigma|=m_{1}^{\sigma}+2 m_{2}^{\sigma}+3 m_{3}^{\sigma}
$$


where $m_{1}^{\sigma}, m_{2}^{\sigma}, m_{3}^{\sigma}$, respectively, are the cardinalities of the sets

$$
\begin{gathered}
\left\{\sigma_{j} \in \sigma \mid 1 \leq \sigma_{j} \leq N\right\}, \\
\left\{\sigma_{j} \in \sigma \mid \sigma_{j}=N+1\right\}, \\
\left\{\sigma_{j} \in \sigma \mid N+2 \leq \sigma_{j} \leq 2 N+1\right\} .
\end{gathered}
$$

Analogously, if $b \in C_{b}^{|\sigma|-2, \alpha}(\Omega)$, we put

$$
D_{\sigma}=D_{\sigma_{1}} \cdots D_{\sigma_{m}}
$$

For greater convenience, whenever we consider a derivative $D_{\sigma}$ with $|\sigma| \geq 2$, in the sequel we agree to assume $b \in C_{b}^{|\sigma|-2, \alpha}(\Omega)$. Moreover, to avoid any ambiguity, when we have a function $F$ which depends on several variables, we systematically write $D(z) F$ instead of $D F(z)$. Then, for instance, $D(z) \Gamma_{\bar{z}}(\cdot, \zeta)$ denotes the $D$-derivative of $\Gamma_{\bar{z}}(\cdot, \zeta)$ evaluated at the point $z$.

The following estimate of $\Gamma_{\bar{z}}$ and its derivatives is well known: for every $\bar{z} \in \Omega_{0}$ and multi-index $\sigma \in\{0,1, \ldots, 2 N+1\}^{m}$ there exists a positive constant $c$, such that

$$
\left|D_{\sigma}^{\bar{z}}(z) \Gamma_{\bar{z}}(\cdot, \zeta)\right| \leq c d_{\bar{z}}(z, \zeta)^{-Q+2-|\sigma|}, \quad \forall z, \zeta \in \Omega_{0}, \quad z \neq \zeta .
$$

Moreover, the constant $c$ in (3.3) depends continuously on $\bar{z}$.

In the proof of Theorem 1.3 we shall also need to compare the fundamental solutions of frozen operators corresponding to different points of $\Omega_{0}$. The main result of this section is the following.

Proposition 3.1. Let $\sigma \in\{1, \ldots, N+1\}^{m}$. There exists a positive constant $c=c\left(\sigma, \Omega_{0}\right)$ such that

$$
\left|D_{\sigma}(z) \Gamma_{\bar{z}}(\cdot, \zeta)-D_{\sigma}(\bar{z}) \Gamma_{\bar{z}}(\cdot, \zeta)\right| \leq c d_{\bar{z}}(\bar{z}, \zeta)^{-Q+2-|\sigma|-\alpha} d_{\bar{z}}(\bar{z}, z)^{\alpha},
$$

and, if $|\sigma| \geq 3$,

$$
\left|D_{\sigma}(z) \Gamma_{z}(\cdot, \zeta)-D_{\sigma}(\bar{z}) \Gamma_{\bar{z}}(\cdot, \zeta)\right| \leq c d_{\bar{z}}(\bar{z}, \zeta)^{-Q+2-|\sigma|-\alpha} d_{\bar{z}}(\bar{z}, z)^{\alpha},
$$

for every $z, \bar{z} \in \Omega_{0}$ and $\zeta \in \Omega_{M}(\bar{z}, z)$ (cf. (2.21) ).

The proof of Proposition 3.1 is based on two lemmas. The first one gives an expression of $D_{j}$ in terms of the frozen vector fields in (2.2).

Lemma 3.2. Let $z, \bar{z} \in \Omega_{0}$ and $\sigma \in\{1, \ldots, N+1\}^{m}$ (and $b \in C_{b}^{|\sigma|-2, \alpha}(\Omega)$, if $|\sigma| \geq 2$ ). For every smooth function $\varphi$, we have

$$
D_{\sigma} \varphi(z)=\sum_{\mu \in\{1, \ldots, N+2\}^{m}} \Lambda_{\mu, \bar{z}}(z)\left(R_{\bar{z}}(z)\right)^{\alpha_{\mu}} D_{\mu}^{\bar{z}} \varphi(z)
$$

where $|\mu| \leq|\sigma|+\alpha_{\mu}, \alpha_{\mu} \leq m_{2}^{\sigma}$, and

$$
\begin{aligned}
R_{\bar{z}}(z) & =\frac{b(z)-P_{\bar{z}}^{1} b(z)}{\partial_{x_{1}} b(\bar{z})}=\frac{b(z)-b(\bar{z})-\left\langle\nabla_{x} b(\bar{z}), x-\bar{x}\right\rangle}{\partial_{x_{1}} b(\bar{z})}, \\
\Lambda_{\mu, \bar{z}}(z) & =\sum_{i} c_{i}(\mu) \prod_{\nu \in J_{\mu, i}}\left(D_{\nu}(z) R_{\bar{z}}\right)^{\beta_{\nu}} .
\end{aligned}
$$

In (3.7), $J_{\mu, i}$ is a suitable subset of $\{1, \ldots, N+1\}^{m-1},|\nu| \leq|\sigma|-2$ and $\beta_{\nu} \leq m$. Moreover, we have

$$
\left|\Lambda_{\mu, \bar{z}}(z)\left(R_{\bar{z}}(z)\right)^{\alpha_{\mu}}-\Lambda_{\mu, \bar{z}}(\bar{z})\left(R_{\bar{z}}(\bar{z})\right)^{\alpha_{\mu}}\right| \leq c d_{\bar{z}}(\bar{z}, z)^{\alpha_{\mu}+\alpha}
$$


for some positive constant $c=c\left(\sigma, \Omega_{0}\right)$.

Proof. We proceed by induction on $|\sigma|$. If $|\sigma|=1,2$, the assertion is trivial since $D_{\sigma}=D_{\sigma}^{\bar{z}}$ if $m_{2}^{\sigma}=0$ (cf. (3.2) ), and we have

$$
D_{N+1}=D_{N+1}^{\bar{z}}+R_{\bar{z}} D_{N+2}^{\bar{z}} \text {. }
$$

We next consider $|\sigma| \geq 2$. If $j=1, \ldots, N$, by induction, we have

$$
\begin{aligned}
D_{j} D_{\sigma} \varphi(z)=\sum_{\mu \in\{1, \ldots, N+2\}^{m}}\left(D_{j}(z)\left(\Lambda_{\mu, \bar{z}}\left(R_{\bar{z}}\right)^{\alpha_{\mu}}\right) D_{\mu}^{\bar{z}} \varphi(z)\right. \\
\left.+\Lambda_{\mu, \bar{z}}(z)\left(R_{\bar{z}}(z)\right)^{\alpha_{\mu}} D_{j}^{\bar{z}} D_{\mu}^{\bar{z}} \varphi(z)\right) .
\end{aligned}
$$

Analogously, we have

$$
\begin{aligned}
& D_{N+1} D_{\sigma} \varphi(z)=\sum_{\mu \in\{1, \ldots, N+2\}^{m}}\left(D_{N+1}(z)\left(\Lambda_{\mu, \bar{z}}\left(R_{\bar{z}}\right)^{\alpha_{\mu}}\right) D_{\mu}^{\bar{z}} \varphi(z)\right. \\
& \left.\quad+\Lambda_{\mu, \bar{z}}(z)\left(R_{\bar{z}}(z)\right)^{\alpha_{\mu}} D_{N+1}^{\bar{z}} D_{\mu}^{\bar{z}} \varphi(z)+\Lambda_{\mu, \bar{z}}(z)\left(R_{\bar{z}}(z)\right)^{\alpha_{\mu}+1} D_{N+2}^{\bar{z}} D_{\mu}^{\bar{z}} \varphi(z)\right) .
\end{aligned}
$$

Then the thesis is a straightforward verification. In particular, 3.8) is a consequence of the fact that, by assumption, $b \in C_{b}^{|\sigma|-2, \alpha}(\Omega)$ and $R_{\bar{z}}(\bar{z})=0$.

The proof of (3.5) in Proposition 3.1 is rather delicate since we need to estimate the fundamental solutions of frozen operators related to different points $z, \bar{z}$. Here we use the canonical change of coordinates (2.5) and we investigate the properties of the fundamental solution $\Gamma_{H}$. The next lemma provides us with some basic estimates.

Lemma 3.3. Let $b \in C_{b}^{1, \alpha}(\Omega)$. There exists a positive constant $c=c(\Omega)$, such that

$$
d_{H}\left(\theta_{\bar{z}}^{\bar{z}}(\zeta), \theta_{z}^{z}(\zeta)\right) \leq c d_{\bar{z}}(\bar{z}, \zeta)^{1-\frac{\alpha}{3}} d_{\bar{z}}(\bar{z}, z)^{\frac{\alpha}{3}},
$$

for every $z, \bar{z} \in \Omega_{0}$ and $\zeta \in \Omega_{M}(\bar{z}, z)$.

Proof. Let us denote $\left(-\theta_{z}^{z}(\zeta)\right) \oplus \theta_{\bar{z}}^{\bar{z}}(\zeta)=\theta$. Keeping in mind formulas (2.5) and (2.6), we get

$$
d_{H}\left(\theta_{\bar{z}}^{\bar{z}}(\zeta), \theta_{z}^{z}(\zeta)\right)=|x-\bar{x}|+|t-\bar{t}|^{\frac{1}{2}}+\sum_{j=N+2}^{2 N+1}\left|\theta_{j}\right|^{\frac{1}{3}} .
$$

If $2 \leq j \leq N$, we have

$$
\begin{aligned}
\theta_{N+1+j} & =\eta_{j}-\bar{y}_{j}+\frac{(\tau-\bar{t})\left(x_{j}+\bar{x}_{j}\right)}{2}-\left(\eta_{j}-y_{j}+\frac{(\tau-t)\left(\xi_{j}+x_{j}\right)}{2}\right) \\
& +\frac{\left(\xi_{j}-x_{j}\right)(\tau-\bar{t})}{2}-\frac{\left(\xi_{j}-x_{j}\right)(\tau-t)}{2} \\
& =\left(y_{j}-\bar{y}_{j}+\frac{(t-\bar{t})\left(x_{j}+\bar{x}_{j}\right)}{2}\right)+\left(\xi_{j}-x_{j}\right)(\tau-\bar{t})-\left(\xi_{j}-\bar{x}_{j}\right)(\tau-t) ;
\end{aligned}
$$

therefore,

$$
\left|\theta_{N+1+j}\right|^{\frac{1}{3}} \leq d_{\bar{z}}(\bar{z}, z)+d_{\bar{z}}(\bar{z}, \zeta)^{\frac{2}{3}} d_{\bar{z}}(\bar{z}, z)^{\frac{1}{3}}+d_{z}(z, \zeta)^{\frac{2}{3}} d_{\bar{z}}(\bar{z}, z)^{\frac{1}{3}}
$$

(since $\zeta \in \Omega_{M}(\bar{z}, z)$ and by (2.22))

$$
\leq c d_{\bar{z}}(\bar{z}, \zeta)^{1-\frac{\alpha}{3}} d_{\bar{z}}(\bar{z}, z)^{\frac{\alpha}{3}} .
$$


Next we prove the inequality

$$
\left|\theta_{N+2}\right|^{\frac{1}{3}} \leq c d_{\bar{z}}(\bar{z}, \zeta)^{1-\frac{\alpha}{3}} d_{\bar{z}}(\bar{z}, z)^{\frac{\alpha}{3}},
$$

which by (3.10) suffices to conclude the proof of the lemma. Noting that

$$
\eta_{j}-y_{j}+x_{j}(\tau-t)=\eta_{j}-y_{j}+\frac{(\tau-t)\left(\xi_{j}+x_{j}\right)}{2}-\frac{(\tau-t)\left(\xi_{j}-x_{j}\right)}{2},
$$

we have

$$
\begin{aligned}
\theta_{N+2}= & \frac{1}{2}(t-\bar{t})\left(\xi_{1}-x_{1}+\xi_{1}-\bar{x}_{1}\right)+\frac{\eta-\bar{y}+(\tau-\bar{t}) b(\bar{z})}{\partial_{x_{1}} b(\bar{z})}-\frac{\eta-y+(\tau-t) b(z)}{\partial_{x_{1}} b(z)} \\
& -\sum_{j=2}^{N}\left[\frac{\partial_{x_{j}} b(\bar{z})}{\partial_{x_{1}} b(\bar{z})}\left(\eta_{j}-\bar{y}_{j}+\bar{x}_{j}(\tau-\bar{t})\right)-\frac{\partial_{x_{j}} b(z)}{\partial_{x_{1}} b(z)}\left(\eta_{j}-y_{j}+x_{j}(\tau-t)\right)\right] \\
(3.13)= & \frac{1}{2}(t-\bar{t})\left(\xi_{1}-x_{1}+\xi_{1}-\bar{x}_{1}\right)+A_{1}+A_{2}+A_{3}+A_{4},
\end{aligned}
$$

where

$$
\begin{aligned}
A_{1}= & \frac{y-\bar{y}+(t-\bar{t}) b(\bar{z})}{\partial_{x_{1}} b(\bar{z})}+\frac{(\tau-t)(b(z)-b(\bar{z}))}{\partial_{x_{1}} b(\bar{z})} \\
& +\frac{\partial_{x_{1}} b(z)-\partial_{x_{1}} b(\bar{z})}{\partial_{x_{1}} b(\bar{z}) \partial_{x_{1}} b(z)}(\eta-y+(\tau-t) b(z)),
\end{aligned}
$$

so that, since $b \in C_{b}^{1, \alpha}(\Omega) \cap C^{1}(\Omega)$, we have

$$
\begin{aligned}
\left|A_{1}\right|^{\frac{1}{3}} \leq & c\left(d_{\bar{z}}(\bar{z}, z)+d_{z}(z, \zeta)^{\frac{2}{3}} d_{\bar{z}}(\bar{z}, z)^{\frac{1}{3}}+d_{\bar{z}}(\bar{z}, z)^{\frac{\alpha}{3}} d_{z}(z, \zeta)\right) \\
& \left(\text { since } \zeta \in \Omega_{M}(\bar{z}, z) \text { and by }(\underline{2.22})\right) \\
\leq & c d_{\bar{z}}(\bar{z}, \zeta)^{1-\frac{\alpha}{3}} d_{\bar{z}}(\bar{z}, z)^{\frac{\alpha}{3}} .
\end{aligned}
$$

Also,

$$
A_{2}=\sum_{j=2}^{N}\left(\frac{\partial_{x_{j}} b(z)}{\partial_{x_{1}} b(z)}-\frac{\partial_{x_{j}} b(\bar{z})}{\partial_{x_{1}} b(\bar{z})}\right)\left(\eta_{j}-\bar{y}_{j}+\bar{x}_{j}(\tau-\bar{t})\right)
$$

so that, since $b \in C_{b}^{1, \alpha}(\Omega)$, we have

$$
\left|A_{2}\right|^{\frac{1}{3}} \leq c d_{\bar{z}}(\bar{z}, z)^{\frac{\alpha}{3}} d_{\bar{z}}(\bar{z}, \zeta) .
$$

Moreover,

$$
A_{3}=\sum_{j=2}^{N} \frac{\partial_{x_{j}} b(z)}{\partial_{x_{1}} b(z)}\left(\eta_{j}-y_{j}+\frac{(\tau-t)\left(x_{j}+x_{j}\right)}{2}-\left(\eta_{j}-\bar{y}_{j}+\frac{(\tau-\bar{t})\left(\xi_{j}+\bar{x}_{j}\right)}{2}\right)\right)
$$

which can be estimated as before:

$$
\left|A_{3}\right|^{\frac{1}{3}} \leq c d_{\bar{z}}(\bar{z}, \zeta)^{1-\frac{\alpha}{3}} d_{\bar{z}}(\bar{z}, z)^{\frac{\alpha}{3}} .
$$

Finally,

$$
\begin{aligned}
A_{4} & =\frac{1}{2} \sum_{j=2}^{N} \frac{\partial_{x_{j}} b(z)}{\partial_{x_{1}} b(z)}\left((\tau-\bar{t})\left(\xi_{j}-\bar{x}_{j}\right)-(\tau-t)\left(\xi_{j}-x_{j}\right)\right) \\
& =\frac{1}{2} \sum_{j=2}^{N} \frac{\partial_{x_{j}} b(z)}{\partial_{x_{1}} b(z)}\left((t-\bar{t})\left(\xi_{j}-\bar{x}_{j}\right)+(\tau-t)\left(x_{j}-\bar{x}_{j}\right)\right)
\end{aligned}
$$


so that

$$
\begin{aligned}
\left|A_{4}\right|^{\frac{1}{3} \leq} \leq & c d_{\bar{z}}(\bar{z}, z)^{\frac{2}{3}} d_{\bar{z}}(\bar{z}, \zeta)^{\frac{1}{3}}+d_{z}(z, \zeta)^{\frac{2}{3}} d_{\bar{z}}(\bar{z}, z)^{\frac{1}{3}} \\
& \left(\text { since } \zeta \in \Omega_{M}(\bar{z}, z) \text { and by }(\underline{2.22})\right) \\
\leq & c d_{\bar{z}}(\bar{z}, \zeta)^{1-\frac{\alpha}{3}} d_{\bar{z}}(\bar{z}, z)^{\frac{\alpha}{3}} .
\end{aligned}
$$

Plugging inequalities (3.14), 3.15), 3.16) and (3.17) back into (3.13), we obtain (3.12). This concludes the proof.

Remark 3.4. Let $b \in C_{b}^{1, \alpha}(\Omega)$. If $z, \bar{z} \in \Omega_{0}, \zeta \in \Omega_{M}(\bar{z}, z)$ and $\theta \in \mathbb{R}^{N+1}$ are such that

$$
d_{H}\left(\theta_{\bar{z}}^{\bar{z}}(\zeta), \theta\right) \leq d_{H}\left(\theta_{\bar{z}}^{\bar{z}}(\zeta), \theta_{z}^{z}(\zeta)\right)
$$

then there exists a positive constant $c=c\left(\Omega_{0}\right)$ such that

$$
\|\theta\|_{H} \geq c\left\|\theta_{\bar{z}}^{\bar{z}}(\zeta)\right\|_{H}
$$

Indeed, we have

$$
\begin{aligned}
d_{\bar{z}}(\bar{z}, \zeta) & =\left\|\theta_{\bar{z}}^{\bar{z}}(\zeta)\right\|_{H} \leq c\left(d_{H}(0, \theta)+d_{H}\left(\theta_{\bar{z}}^{\bar{z}}(\zeta), \theta\right)\right) \\
& \leq\left(d_{H}(0, \theta)+d_{H}\left(\theta_{\bar{z}}^{\bar{z}}(\zeta), \theta_{z}^{z}(\zeta)\right)\right)
\end{aligned}
$$

(by (3.9) and since $\zeta \in \Omega_{M}(\bar{z}, z)$ )

$$
\leq c\left(d_{H}(0, \theta)+M^{-\frac{\alpha}{3}} d_{\bar{z}}(\bar{z}, \zeta)\right) .
$$

Thus, if $M$ is suitably large, we get (3.18).

We are now in position to prove Proposition 3.1

Proof of Proposition 3.1. We first prove estimate (3.4) by using Lemma 3.2. We have

$$
\left|\left(D_{\sigma}(z)-D_{\sigma}(\bar{z})\right) \Gamma_{\bar{z}}(\cdot, \zeta)\right| \leq A_{1}+A_{2},
$$

where

$$
A_{1}=\sum_{\mu \in\{1, \ldots, N+2\}^{m}}\left|\Lambda_{\mu, \bar{z}}(z)\left(R_{\bar{z}}(z)\right)^{\alpha_{\mu}}-\Lambda_{\mu, \bar{z}}(\bar{z})\left(R_{\bar{z}}(\bar{z})\right)^{\alpha_{\mu}}\right|\left|D_{\mu}^{\bar{z}}(\bar{z}) \Gamma_{\bar{z}}(\cdot, \zeta)\right|
$$

and

$$
A_{2}=\sum_{\mu \in\{1, \ldots, N+2\}^{m}}\left|\Lambda_{\mu, \bar{z}}(z)\left(R_{\bar{z}}(z)\right)^{\alpha_{\mu}}\left(D_{\mu}^{\bar{z}}(z)-D_{\mu}^{\bar{z}}(\bar{z})\right) \Gamma_{\bar{z}}(\cdot, \zeta)\right| .
$$

Hence, by (3.3) and (3.8), we have

$$
A_{1} \leq c \sum_{\mu \in\{1, \ldots, N+2\}^{m}} d_{\bar{z}}(\bar{z}, z)^{\alpha_{\mu}+\alpha} d_{\bar{z}}(\bar{z}, \zeta)^{-Q+2-|\mu|}
$$

(since $\zeta \in \Omega_{M}(\bar{z}, z)$ and, by Lemma (3.2), $|\mu| \leq|\sigma|+\alpha_{\mu}$ )

$$
\leq c d_{\bar{z}}(\bar{z}, z)^{\alpha} d_{\bar{z}}(\bar{z}, \zeta)^{-Q+2-|\sigma|} .
$$


On the other hand, by the mean value theorem, for some $\widetilde{z}$ such that $d_{\bar{z}}(\bar{z}, \widetilde{z}) \leq$ $d_{\bar{z}}(\bar{z}, z)$, we have

$$
\begin{aligned}
\left|\left(D_{\mu}^{\bar{z}}(z)-D_{\mu}^{\bar{z}}(\bar{z})\right) \Gamma_{\bar{z}}(\cdot, \zeta)\right| & =\left|\left\langle\theta_{\bar{z}}^{\bar{z}}(z), \nabla_{\bar{z}} D_{\mu}^{\bar{z}}(\widetilde{z}) \Gamma_{\bar{z}}(\cdot, \zeta)\right\rangle\right| \\
& \leq c \sum_{j=1}^{3} d_{\bar{z}}(\bar{z}, z)^{j} d_{\bar{z}}(\bar{z}, \zeta)^{-Q+2-|\mu|-j}
\end{aligned}
$$

(since $\left.\zeta \in \Omega_{M}(\bar{z}, z)\right)$

$$
\leq c d_{\bar{z}}(\bar{z}, \zeta)^{-Q+2-|\mu|-\alpha} d_{\bar{z}}(\bar{z}, z)^{\alpha} .
$$

As before, we conclude that

$$
A_{2} \leq c d_{\bar{z}}(\bar{z}, \zeta)^{-Q+2-|\sigma|-\alpha} d_{\bar{z}}(\bar{z}, z)^{\alpha} .
$$

Next we prove (3.5). By (3.1) and Lemma 3.2, we have

$$
\begin{aligned}
& \left|D_{\sigma}(z) \Gamma_{z}(\cdot, \zeta)-D_{\sigma}(\bar{z}) \Gamma_{\bar{z}}(\cdot, \zeta)\right| \\
& \leq\left|\sum_{|\mu| \leq|\sigma|}\left(\frac{\Lambda_{\mu, z}(z)}{\partial_{x_{1}} b(z)} D_{\mu}^{H}\left(-\theta_{z}^{z}(\zeta)\right)-\frac{\Lambda_{\mu, \bar{z}}(\bar{z})}{\partial_{x_{1}} b(\bar{z})} D_{\mu}^{H}\left(-\theta_{\bar{z}}^{\bar{z}}(\zeta)\right)\right) \Gamma_{H}\right| \leq A_{1}+A_{2}
\end{aligned}
$$

where

$$
\begin{aligned}
& A_{1}=\sum_{|\mu| \leq|\sigma|}\left|\frac{\Lambda_{\mu, z}(z)}{\partial_{x_{1}} b(z)}-\frac{\Lambda_{\mu, \bar{z}}(\bar{z})}{\partial_{x_{1}} b(\bar{z})}\right|\left|D_{\mu}^{H} \Gamma_{H}\left(-\theta_{z}^{z}(\zeta)\right)\right| \\
& A_{2}=\sum_{|\mu| \leq|\sigma|}\left|\frac{\Lambda_{\mu, \bar{z}}(\bar{z})}{\partial_{x_{1}} b(\bar{z})}\right|\left|D_{\mu}^{H} \Gamma_{H}\left(-\theta_{z}^{z}(\zeta)\right)-D_{\mu}^{H} \Gamma_{H}\left(-\theta_{\bar{z}}^{\bar{z}}(\zeta)\right)\right|
\end{aligned}
$$

Since the function

$$
z \longmapsto \frac{\Lambda_{\mu, z}(z)}{\partial_{x_{1}} b(z)} \in C_{B}^{\alpha}\left(\Omega_{0}\right)
$$

we get

$$
A_{1} \leq c d_{\bar{z}}(\bar{z}, z)^{\alpha} d_{z}(z, \zeta)^{-Q+2-|\sigma|} .
$$

On the other hand, by the mean value theorem, there exists $\theta$ such that

$$
d_{H}\left(\theta_{\bar{z}}^{\bar{z}}(\zeta), \theta\right) \leq d_{H}\left(\theta_{\bar{z}}^{\bar{z}}(\zeta), \theta_{z}^{z}(\zeta)\right)
$$

and

$$
\begin{aligned}
\left|D_{\mu}^{H} \Gamma_{H}\left(-\theta_{z}^{z}(\zeta)\right)-D_{\mu}^{H} \Gamma_{H}\left(-\theta_{\bar{z}}^{\bar{z}}(\zeta)\right)\right| & =\left|\left\langle\left(-\theta_{z}^{z}(\zeta)\right) \oplus \theta_{\bar{z}}^{\bar{z}}(\zeta), \nabla_{H} D_{\mu}^{H} \Gamma_{H}(\theta)\right\rangle\right| \\
& \leq c \sum_{j=1}^{3} d_{H}\left(\theta_{\bar{z}}^{\bar{z}}(\zeta), \theta_{z}^{z}(\zeta)\right)^{j}\|\theta\|_{H}^{-Q+2-|\mu|-j} \\
& \leq c d_{\bar{z}}(\bar{z}, z)^{\alpha} d_{z}(z, \zeta)^{-Q+2-|\sigma|-\alpha},
\end{aligned}
$$

where the last inequality follows from Lemma 3.3 and Remark 3.4 (note that the assumption $b \in C_{b}^{1, \alpha}(\Omega)$ of Lemma 3.3 is fulfilled because $\left.|\sigma| \geq 3\right)$. The proof is completed.

We end this section by describing the fine behaviour of the vector fields $D_{j}^{\bar{z}}$ through the right translations w.r.t. the law "o" in (2.11). 
Lemma 3.5. Let $b \in C_{b}^{1, \alpha}(\Omega)$. For every smooth function $\varphi$ and multi-index $\mu \in\{1, \ldots, N+2\}^{m}$, we have

$$
D_{\mu}^{\bar{z}}(z) \varphi\left(\cdot \circ \zeta^{-1}\right)=\sum_{\nu \in\{1, \ldots, 2 N+1\}^{m}} P_{\nu, \bar{z}}(\zeta) D_{\nu}^{\bar{z}}\left(z \circ \zeta^{-1}\right) \varphi,
$$

where $P_{\nu, \bar{z}}$ is a polynomial $\delta_{\lambda}^{\bar{z}}$-homogeneous of degree $k_{\nu}$, with $|\nu| \leq|\mu|+k_{\nu}$, whose coefficients are functions of $\bar{z}$ of class $C_{B}^{\alpha}\left(\Omega_{0}\right)$.

Proof. Since

$$
z \circ \zeta^{-1}=\left(x-\xi, y-\eta+\mathcal{J}_{x} B(\bar{z})(\tau(x-\bar{x})-(\tau-\bar{t}) \xi), t-\tau\right),
$$

we have, for $2 \leq j \leq N$,

$$
\begin{aligned}
D_{1}^{\bar{z}}(z) \varphi\left(\cdot \circ \zeta^{-1}\right) & =D_{1}^{\bar{z}}\left(z \circ \zeta^{-1}\right) \varphi+\tau D_{N+2}^{\bar{z}}\left(z \circ \zeta^{-1}\right) \varphi \\
D_{j}^{\bar{z}}(z) \varphi\left(\cdot \circ \zeta^{-1}\right) & =D_{j}^{\bar{z}}\left(z \circ \zeta^{-1}\right) \varphi+\tau\left(D_{N+1+j}^{\bar{z}}-\frac{1}{\partial_{x_{1}} b(\bar{z})} D_{N+2}^{\bar{z}}\right)\left(z \circ \zeta^{-1}\right) \varphi \\
D_{N+1}^{\bar{z}}(z) \varphi\left(\cdot \circ \zeta^{-1}\right) & =\left(D_{N+1}^{\bar{z}}+\sum_{j=2}^{N} \xi_{j} D_{N+1+j}^{\bar{z}}\right)\left(z \circ \zeta^{-1}\right) \varphi \\
D_{N+2}^{\bar{z}}(z) \varphi\left(\cdot \circ \zeta^{-1}\right) & =D_{N+2}^{\bar{z}}\left(z \circ \zeta^{-1}\right) \varphi .
\end{aligned}
$$

The thesis easily follows by induction on $|\mu|$.

\section{HÖLDER REGULARITY}

In this section we prove Theorem 1.3 We first give the definition of a classical solution of (1.1).

Definition 4.1. A classical solution of (1.1) is a function $u \in C^{1}(\Omega)$, with secondorder derivatives $\partial_{x_{i} x_{j}} u \in C(\Omega), 1 \leq i, j \leq N$, verifying (1.1).

For greater convenience, since in the following proof we deal with several estimates, we shall denote by $c$ a constant which will not be always the same.

Proof of Theorem 1.3. We proceed by induction on $k$. Let us remark that, if $k \geq 7$, the proof is standard and the thesis follows by differentiating the equation. More precisely, if $b, f \in C_{b}^{k-2, \alpha}(\Omega)$, with $0<\alpha<1$ and $k \geq 7$, then by the inductive hypothesis, $u \in C_{b}^{k-1, \bar{\alpha}}(\Omega)$ for every $\left.\bar{\alpha} \in\right] 0, \alpha$. Subsequently, by differentiating equation (1.1) w.r.t. the variable $y$ and by denoting, as usual, $\partial_{y} u=u_{y}$, we get

$$
\left.L_{b} u_{y}=f_{y}-b_{y} u_{y} \in C_{b}^{k-5, \bar{\alpha}}(\Omega), \forall \bar{\alpha} \in\right] 0, \alpha[.
$$

Thus, we deduce that $u_{y} \in C_{b}^{k-3, \bar{\alpha}}(\Omega)$. Next we differentiate equation (1.1) w.r.t. the variable $x_{j}, j=1, \ldots, N-1$, and we get

$$
\left.L_{b} u_{x_{j}}=f_{x_{j}}-b_{x_{j}} u_{y} \in C_{b}^{k-3, \bar{\alpha}}(\Omega), \forall \bar{\alpha} \in\right] 0, \alpha[.
$$

Therefore, we deduce that $u_{x_{j}} \in C_{b}^{k-1, \bar{\alpha}}(\Omega)$. Finally, we differentiate equation (1.1) w.r.t. to $X_{N+1}=b \partial_{y}-\partial_{t}$ :

$$
\left.L_{b}\left(X_{N+1} u\right)=X_{N+1} f+2\left\langle\nabla_{x} b, \nabla_{x} u_{y}\right\rangle+u_{y} \Delta_{x} b \in C_{b}^{k-4, \bar{\alpha}}(\Omega), \forall \bar{\alpha} \in\right] 0, \alpha[.
$$

Hence, $X_{N+1} u \in C_{b}^{k-2, \bar{\alpha}}(\Omega)$ and this proves that $u \in C_{b}^{k, \bar{\alpha}}(\Omega)$ for every $\left.\bar{\alpha} \in\right] 0, \alpha[$.

We next consider $3 \leq k \leq 6$. We set the problem in dimension $2 N+1$ and we prove that $u \in C_{B}^{k, \bar{\alpha}}\left(\Omega_{0}\right)$. Then, by Remark[2.5, we infer that $u \in C_{b}^{k, \bar{\alpha}}(\Omega)$. We split 
the proof into two steps: existence of the derivatives and Hölder continuity. Since the case $k=2$ is easier, we shall only sketch its proof separately at the end.

The case $3 \leq k \leq 6$ : existence of the derivatives. Let $b, f \in C_{b}^{k-2, \alpha}(\Omega)$, with $0<\alpha<1$ and $3 \leq k \leq 6$. By the inductive hypothesis, $u \in C_{b}^{k-1, \bar{\alpha}}(\Omega)$ for every $\bar{\alpha} \in] 0, \alpha[$. Since we aim to prove a local result, we only prove the existence of the derivatives of order $k$ of $u$ in a domain $E_{0}$, where $\bar{E}_{0}$ is a compact subset of $\Omega_{0}$. To this end, we represent the solution $u$ in terms of the fundamental solution $\Gamma_{\bar{z}}$, $\bar{z} \in E_{0}$. More precisely, we consider a cut-off function $\varphi \in C_{0}^{\infty}\left(\Omega_{0}\right)$ such that $\varphi=1$ in a neighborhood of $E_{0}$. We remark that it is not restrictive to assume that

$$
\operatorname{supp}(\nabla \varphi) \subseteq \Omega_{M}(\bar{z}, z), \quad \forall z, \bar{z} \in E_{0},
$$

where $M$ is large so that $(2.22)$ holds for every $z, \bar{z} \in E_{0}$. We have

$$
\begin{aligned}
(u \varphi)(z) & =\int_{\Omega_{0}} \Gamma_{\bar{z}}(z, \zeta) L_{\bar{z}}(u \varphi)(\zeta) d \zeta \\
& =\int_{\Omega_{0}} \Gamma_{\bar{z}}(z, \zeta)\left(\varphi\left(f-\left(b-P_{\bar{z}}^{1} b\right) u_{y_{1}}\right)+2\left\langle\nabla_{x} u, \nabla_{x} \varphi\right\rangle+u L_{\bar{z}} \varphi\right) d \zeta
\end{aligned}
$$

Consequently, the solution $u$ can be expressed in the form

$$
u(z)=I_{1, \bar{z}}(z)-I_{2, \bar{z}}(z), \quad z \in E_{0}
$$

where

$$
I_{j, \bar{z}}(z)=\int_{\Omega_{0}} \Gamma_{\bar{z}}(z, \zeta) U_{j, \bar{z}}(\zeta) d \zeta, \quad j=1,2,
$$

with $U_{1, \bar{z}} \in C_{0}^{\infty}\left(\Omega_{0}\right)$ and

$$
\left.\left|U_{2, \bar{z}}(\zeta)\right| \leq c d_{\bar{z}}(\bar{z}, \zeta)^{k-2+\bar{\alpha}}, \quad \forall \bar{z} \in E_{0}, \zeta \in \Omega_{0}, \bar{\alpha} \in\right] 0, \alpha[.
$$

Indeed, it suffices to put

$$
\begin{aligned}
U_{1, \bar{z}}= & \varphi\left(P_{\bar{z}}^{k-2} f+P_{\bar{z}}^{k-4} u_{y_{1}}\left(P_{\bar{z}}^{k-2} b-P_{\bar{z}}^{1} b\right)\right) \\
& +2\left\langle P_{\bar{z}}^{k-2} \nabla_{x} u, \nabla_{x} \varphi\right\rangle+\left(L_{\bar{z}} \varphi\right) P_{\bar{z}}^{k-2} u \\
U_{2, \bar{z}}= & \varphi\left(u_{y_{1}}\left(b-P_{\bar{z}}^{k-2} b\right)+\left(P_{\bar{z}}^{k-2} b-P_{\bar{z}}^{1} b\right)\left(u_{y_{1}}-P_{\bar{z}}^{k-4} u_{y_{1}}\right)+f-P_{\bar{z}}^{k-2} f\right) \\
& +2\left\langle\nabla_{x} u-P_{\bar{z}}^{k-2} \nabla_{x} u, \nabla_{x} \varphi\right\rangle+L_{\bar{z}} \varphi\left(u-P_{\bar{z}}^{k-2} u\right)
\end{aligned}
$$

where we agree that $P_{\bar{z}}^{h} u \equiv 0$ if $h<0$. Hence, (4.3) is a consequence of the regularity assumptions on $u, b, f$ and of the estimate

$$
P_{\bar{z}}^{k-2} b(\zeta)-P_{\bar{z}}^{1} b(\zeta)=O\left(d_{\bar{z}}(\bar{z}, \zeta)^{2}\right), \quad \text { as } \zeta \longrightarrow \bar{z},
$$

which can be easily deduced from the homogeneity property of the Taylor polynomial $P_{\bar{z}}^{h} b$. Note that $I_{1, \bar{z}}$ is a smooth function on $E_{0}$. Indeed, by the change of variables $\bar{\zeta}=\zeta^{-1} \circ z$, we have

$$
I_{1, \bar{z}}(z)=\int \Gamma_{\bar{z}}(\bar{\zeta}, 0) U_{1, \bar{z}}\left(z \circ \bar{\zeta}^{-1}\right) d \bar{\zeta}
$$

and $U_{1, \bar{z}} \in C_{0}^{\infty}\left(\Omega_{0}\right)$.

On the other hand, the function

$$
J_{\sigma}(\bar{z})=\int_{\Omega_{0}} D_{\sigma}(\bar{z}) \Gamma_{\bar{z}}(\cdot, \zeta) U_{2, \bar{z}}(\zeta) d \zeta, \quad \bar{z} \in E_{0},
$$


is well defined for $|\sigma|=k$. Indeed, by Lemma 3.2, we have

$$
\left|D_{\sigma}(\bar{z}) \Gamma_{\bar{z}}(\cdot, \zeta)\right|=\left|\sum_{|\mu| \leq|\sigma|} \Lambda_{\mu, \bar{z}}(\bar{z}) D_{\mu}^{\bar{z}}(\bar{z}) \Gamma_{\bar{z}}(\cdot, \zeta)\right| \leq c d_{\bar{z}}(\bar{z}, \zeta)^{-Q+2-|\sigma|} ;
$$

therefore, by (4.3), the integral in the right-hand side of (4.7) converges.

We claim that, for every multi-index $\sigma \in\{1, \ldots, N+1\}^{m}$, with $|\sigma| \leq k$, we have

$$
D_{\sigma} u(\bar{z})=D_{\sigma}(\bar{z}) I_{1, \bar{z}}-J_{\sigma}(\bar{z}), \quad \forall \bar{z} \in E_{0} .
$$

The proof of (4.9) is based on the use of some suitable high-order difference quotients related to the system $D_{j}, j=1, \ldots, N+1$. For $z \in \Omega$ and $\delta \in \mathbb{R} \backslash\{0\}$ sufficiently small, we set

$$
\begin{aligned}
\triangle_{(j)}(u, z, \delta) & =\frac{u\left(\exp \left(\delta D_{j}\right)(z)\right)-u(z)}{\delta}, \quad 1 \leq j \leq N, \\
\triangle_{(N+1)}(u, z, \delta) & =\frac{u\left(\exp \left(\delta^{2} D_{N+1}\right)(z)\right)-u(z)}{\delta^{2}} .
\end{aligned}
$$

Also, if $\sigma \in\{1, \ldots, N+1\}^{m}$, by recurrence we define

$$
\triangle_{\sigma}(u, z, \delta)=\triangle_{\left(\sigma_{m}\right)}\left(\triangle_{\left(\sigma_{1}, \ldots, \sigma_{m-1}\right)}(u, \cdot, \delta), z, \delta\right) .
$$

The following result can be proved as in [6], Remark 4.2.

Lemma 4.2. Let $u \in C_{b}^{k-1, \alpha}(\Omega)$ and $\sigma \in\{1, \ldots, N+1\}^{m},|\sigma|=k$. If there exists

$$
\lim _{\delta \rightarrow 0} \triangle_{\sigma}(u, \cdot, \delta)=v
$$

uniformly on compact subsets of $\Omega$, then there exists $D_{\sigma} u=v$.

We are now in a position to prove (4.9). Since $I_{1, \bar{z}}$ is a smooth function, by the mean value theorem, we have

$$
\triangle_{\sigma}\left(I_{1, \bar{z}}, \bar{z}, \delta\right)=D_{\sigma}\left(z_{\delta}\right) I_{\bar{z}}^{1}
$$

for some $z_{\delta}$ such that

$$
d_{\bar{z}}\left(\bar{z}, z_{\delta}\right) \leq c_{1} \delta,
$$

where the constant $c_{1}$ depends only on $\sigma$ and on the constant $c_{0}$ in 2.16) (for instance, $c_{1}=m c_{0}^{m-1}$ is fine). Thus, $\triangle_{\sigma}\left(I_{1, \bar{z}}, \bar{z}, \delta\right)$ converges to $D_{\sigma}(\bar{z}) I_{1, \bar{z}}$ as $\delta$ tends to zero uniformly in $\bar{z} \in E_{0}$. Therefore, by Lemma 4.2, in order to prove (4.9), it suffices to show that

$$
\lim _{\delta \rightarrow 0} \triangle_{\sigma}\left(I_{2, \bar{z}}, \bar{z}, \delta\right)=J_{\sigma}(\bar{z})
$$

uniformly on $E_{0}$.

Let us consider a cut-off function $\chi \in C_{0}^{\infty}(\mathbb{R},[0,1])$ such that $\chi(s)=1$ for $s \geq 2$ and $\chi$ vanishes for $s \leq 1$. We set

$$
I_{2, \bar{z}, \delta}(z)=\int_{\Omega_{0}} \Gamma_{\bar{z}}(z, \zeta) \chi\left(\frac{d_{\bar{z}}(\bar{z}, \zeta)}{2 c_{1} M \delta}\right) U_{2, \bar{z}}(\zeta) d \zeta, \quad z \in E_{0},
$$

where $M, c_{1}$ are the constants in (4.1), (4.10) respectively. Note that

$$
z \longmapsto I_{2, \bar{z}, \delta}(z), \quad d_{\bar{z}}(\bar{z}, z) \leq c_{1} \delta,
$$


is a smooth function. Indeed, if $d_{\bar{z}}(\bar{z}, z) \leq c_{1} \delta$, then by (2.16), the argument of the cut-off function $\chi$ in (4.12) satisfies

$$
\frac{d_{\bar{z}}(\bar{z}, \zeta)}{2 c_{1} M \delta} \leq \frac{1}{2 c_{1} \delta}\left(d_{\bar{z}}(\bar{z}, z)+d_{z}(z, \zeta)\right) \leq 1, \quad \forall \zeta, d_{z}(z, \zeta) \leq c_{1} \delta,
$$

so that $\chi$ vanishes in a neighborhood of the pole of $\Gamma_{\bar{z}}(z, \zeta)$.

We claim that

$$
\begin{array}{r}
\sup _{d_{\bar{z}}(\bar{z}, z) \leq c_{1} \delta}\left|I_{2, \bar{z}, \delta}(z)-I_{2, \bar{z}}(z)\right| \leq c \delta^{k+\bar{\alpha}}, \\
\sup _{d_{\bar{z}}(\bar{z}, z) \leq c_{1} \delta}\left|D_{\sigma}(z) I_{2, \bar{z}, \delta}-J_{\sigma}(\bar{z})\right| \leq c \delta^{\bar{\alpha}},
\end{array}
$$

for some positive constant $c$ which depends continuously on $\bar{z}$. Taking the claim for granted, we immediately conclude the proof of (4.11) (and consequently of (4.9)):

$$
\left|\triangle_{\sigma}\left(I_{2, \bar{z}}, \bar{z}, \delta\right)-J_{\sigma}(\bar{z})\right|
$$

(by the mean value theorem, for some $z_{\delta}$ such that $d_{\bar{z}}\left(\bar{z}, z_{\delta}\right) \leq c_{1} \delta$ )

$$
\leq\left|\triangle_{\sigma}\left(I_{2, \bar{z}}, \bar{z}, \delta\right)-\triangle_{\sigma}\left(I_{2, \bar{z}, \delta}, \bar{z}, \delta\right)\right|+\left|D_{\sigma}\left(z_{\delta}\right) I_{2, \bar{z}, \delta}-J_{\sigma}(\bar{z})\right|
$$

(by 4.13) and (4.14))

$$
\leq c \delta^{\bar{\alpha}} .
$$

We are left with the proof of the claim. We begin by proving (4.13). We have

$$
\left|I_{2, \bar{z}}(z)-I_{2, \bar{z}, \delta}(z)\right| \leq \int_{d_{\bar{z}}(\bar{z}, \zeta) \leq 4 c_{1} M \delta} \Gamma_{\bar{z}}(z, \zeta)\left|U_{2, \bar{z}}(\zeta)\right| d \zeta
$$

(by (3.3) and (4.3))

$$
\leq c \delta^{k-2+\bar{\alpha}} \int_{d_{\bar{z}}(\bar{z}, \zeta) \leq 4 c_{1} M \delta} d_{\bar{z}}(z, \zeta)^{-Q+2} d \zeta
$$

(since, by assumption, $\left.d_{\bar{z}}(z, \zeta) \leq c_{0}\left(d_{\bar{z}}(\bar{z}, z)+d_{\bar{z}}(\bar{z}, \zeta)\right) \leq c_{0}\left(c_{1} \delta+d_{\bar{z}}(\bar{z}, \zeta)\right)\right)$

$$
\leq c \delta^{k-2+\bar{\alpha}} \int_{d_{\bar{z}}(z, \zeta) \leq c_{0} c_{1}(1+4 M) \delta} d_{\bar{z}}(z, \zeta)^{-Q+2} d \zeta \leq c \delta^{k+\bar{\alpha}} .
$$

Next, we prove (4.14). We have

$$
\left|D_{\sigma}(z) I_{2, \bar{z}, \delta}-J_{\sigma}(\bar{z})\right| \leq A_{1}(\bar{z}, z)+A_{2}(\bar{z}, z),
$$

where

$$
A_{1}(\bar{z}, z)=\int_{\Omega_{0}}\left|D_{\sigma}(\bar{z}) \Gamma_{\bar{z}}(\cdot, \zeta)\right|\left(1-\chi\left(\frac{d_{\bar{z}}(\bar{z}, \zeta)}{2 c_{1} M \delta}\right)\right)\left|U_{2, \bar{z}}(\zeta)\right| d \zeta
$$

and

$$
A_{2}(\bar{z}, z)=\int_{\Omega_{0}}\left|\left(D_{\sigma}(z)-D_{\sigma}(\bar{z})\right) \Gamma_{\bar{z}}(\cdot, \zeta)\right| \chi\left(\frac{d_{\bar{z}}(\bar{z}, \zeta)}{2 c_{1} M \delta}\right)\left|U_{2, \bar{z}}(\zeta)\right| d \zeta
$$

Proceeding as in the proof of (4.13) and using (4.8), we obtain

$$
A_{1}(\bar{z}, z) \leq c \delta^{\bar{\alpha}} .
$$


On the other hand, we remark that, if $d_{\bar{z}}(\bar{z}, z) \leq c_{1} \delta$, then

$$
\operatorname{supp}\left(\chi\left(\frac{d_{\bar{z}}(\bar{z}, \cdot)}{2 c_{1} M \delta}\right)\right) \subseteq \Omega_{M}
$$

Hence, by estimate (3.4) of Proposition 3.1 and (4.3), we get

$$
A_{2}(\bar{z}, z) \leq c \int_{\Omega_{M}} d_{\bar{z}}(\bar{z}, z)^{\bar{\alpha}} d_{\bar{z}}(\bar{z}, \zeta)^{-Q+\bar{\alpha}} d \zeta \leq c \delta^{\bar{\alpha}} .
$$

This concludes the proof of (4.14).

The case $3 \leq k \leq 6$ : Hölder continuity of the derivatives. Let $\alpha \in] 0,1[$ and $3 \leq k \leq 6$. In the previous step, we have proved that, if $b, f \in C_{b}^{k-2, \alpha}(\Omega)$, and $u \in C_{b}^{k-1, \bar{\alpha}}(\Omega)$, for any $\left.\bar{\alpha} \in\right] 0, \alpha\left[\right.$, then $u$ is $D_{\sigma}$-differentiable for any $\sigma$ of weight $|\sigma| \leq k$ and the representation formula (4.9) of $D_{\sigma} u$ holds.

In this step, we aim to prove that

$$
\begin{array}{lr}
D_{\sigma} u \in C_{B}^{\bar{\alpha}}\left(E_{0}\right), & \left.\forall \sigma \in\{1, \ldots, N+1\}^{m},|\sigma|=k, \text { and } \bar{\alpha} \in\right] 0, \alpha[, \\
D_{\sigma} u \in C_{D_{N+1}}^{1+\bar{\alpha}}\left(E_{0}\right), & \forall \sigma,|\sigma|=k-1, \text { and } \bar{\alpha} \in] 0, \alpha[,
\end{array}
$$

where $E_{0}$ is any domain fixed as in the preceding step. As a consequence of (4.15) and (4.16), we deduce that $u \in C_{b}^{k, \bar{\alpha}}(\Omega)$.

We prove (4.15) by means of Lemma 2.8 and we consider separately the terms $D_{\sigma}(\bar{z}) I_{1, \bar{z}}$ and $J_{\sigma}(\bar{z})$ in formula (4.9), with $\bar{z} \in E_{0}$ and $|\sigma|=k$. Let us begin with $J_{\sigma}$ and prove that

$$
\left.\left|J_{\sigma}(z)-J_{\sigma}(\bar{z})\right| \leq c d_{\bar{z}}(\bar{z}, z)^{\bar{\alpha}}, \quad \forall \bar{z}, z \in E_{0}, \bar{\alpha} \in\right] 0, \alpha[,
$$

for some constant $c=c\left(E_{0}, \bar{\alpha}, k\right)$. By a standard decomposition, we obtain

$$
J_{\sigma}(z)-J_{\sigma}(\bar{z})=A_{1}(z, \bar{z})+A_{2}(z, \bar{z})+A_{3}(z, \bar{z}),
$$

where

$$
\begin{aligned}
& A_{1}(z, \bar{z})=\int_{\Omega_{0} \backslash \Omega_{M}(\bar{z}, z)}\left(D_{\sigma}(z) \Gamma_{z}(\cdot, \zeta) U_{2, z}(\zeta)-D_{\sigma}(\bar{z}) \Gamma_{\bar{z}}(\cdot, \zeta) U_{2, \bar{z}}(\zeta)\right) d \zeta, \\
& A_{2}(z, \bar{z})=\int_{\Omega_{M}(\bar{z}, z)}\left(D_{\sigma}(z) \Gamma_{z}(\cdot, \zeta)-D_{\sigma}(\bar{z}) \Gamma_{\bar{z}}(\cdot, \zeta)\right) U_{2, \bar{z}}(\zeta) d \zeta, \\
& A_{3}(z, \bar{z})=\int_{\Omega_{M}(\bar{z}, z)} D_{\sigma}(z) \Gamma_{z}(\cdot, \zeta)\left(U_{2, z}(\zeta)-U_{2, \bar{z}}(\zeta)\right) d \zeta .
\end{aligned}
$$

By (4.3) and (4.8), it is straightforward to prove that

$$
\left|A_{1}(z, \bar{z})\right| \leq c d_{\bar{z}}(\bar{z}, z)^{\bar{\alpha}}, \quad \forall \bar{z}, z \in E_{0} .
$$

Next, we estimate $A_{2}(z, \bar{z})$ by using (3.5) of Proposition 3.1. We deduce that, for every $\overline{\bar{\alpha}} \in] 0, \bar{\alpha}\left[\right.$, there exists a constant $c=c\left(E_{0}, \overline{\bar{\alpha}}\right)$, such that

$$
\left|A_{2}(z, \bar{z})\right| \leq c \int_{\Omega_{M}(\bar{z}, z)} d_{\bar{z}}(\bar{z}, \zeta)^{-Q} d_{\bar{z}}(\bar{z}, z)^{\bar{\alpha}} d \zeta \leq c d_{\bar{z}}(\bar{z}, z)^{\overline{\bar{\alpha}}}, \quad \forall \bar{z}, z \in E_{0} .
$$

Analogously, we have

$$
\left|A_{3}(z, \bar{z})\right| \leq c d_{\bar{z}}(\bar{z}, z)^{\overline{\bar{\alpha}}}, \quad \forall \bar{z}, z \in E_{0} .
$$


Indeed, (4.18) is an immediate consequence of (4.8) and of the following estimate: there exists a positive constant $c=c\left(E_{0}\right)$ such that

$$
\left|U_{2, z}(\zeta)-U_{2, \bar{z}}(\zeta)\right| \leq c d_{\bar{z}}(\bar{z}, z)^{\bar{\alpha}} d_{\bar{z}}(\bar{z}, \zeta)^{k-2}, \quad \forall z, \bar{z} \in E_{0}, \zeta \in \Omega_{M}(\bar{z}, z)
$$

Let us prove (4.19). We have

$$
U_{2, z}-U_{2, \bar{z}}=B_{1}(z, \bar{z})+\varphi B_{2}(z, \bar{z})+B_{3}(z, \bar{z}),
$$

where

$$
\begin{aligned}
B_{1}(z, \bar{z})= & \varphi\left(u_{y_{1}}\left(P_{\bar{z}}^{k-2} b-P_{z}^{k-2} b\right)+\left(P_{\bar{z}}^{k-2} f-P_{z}^{k-2} f\right)\right) \\
& +2\left\langle P_{\bar{z}}^{k-2} \nabla_{x} u-P_{z}^{k-2} \nabla_{x} u, \nabla_{x} \varphi\right\rangle \\
B_{2}(z, \bar{z})= & \left(P_{z}^{k-2} b-P_{z}^{1} b\right)\left(u_{y_{1}}-P_{z}^{k-4} u_{y_{1}}\right)-\left(P_{\bar{z}}^{k-2} b-P_{\bar{z}}^{1} b\right)\left(u_{y_{1}}-P_{\bar{z}}^{k-4} u_{y_{1}}\right) \\
B_{3}(z, \bar{z})= & L_{z} \varphi\left(u-P_{z}^{k-2} u\right)-L_{\bar{z}} \varphi\left(u-P_{\bar{z}}^{k-2} u\right)
\end{aligned}
$$

By (2.23) of Lemma 2.10, we get at once

$$
\left|B_{1}(z, \bar{z})(\zeta)\right| \leq c d_{\bar{z}}(\bar{z}, z)^{\bar{\alpha}} d_{\bar{z}}(\bar{z}, \zeta)^{k-2}, \quad \forall z, \bar{z} \in E_{0}, \zeta \in \Omega_{M}(\bar{z}, z) .
$$

We next remark that

$$
\begin{aligned}
B_{2}(z, \bar{z}) & =\left(P_{z}^{k-2} b-P_{z}^{1} b\right)\left(P_{\bar{z}}^{k-4} u_{y_{1}}-P_{z}^{k-4} u_{y_{1}}\right) \\
& +\left[\left(P_{z}^{k-2} b-P_{z}^{1} b\right)-\left(P_{\bar{z}}^{k-2} b-P_{\bar{z}}^{1} b\right)\right]\left(u_{y_{1}}-P_{\bar{z}}^{k-4} u_{y_{1}}\right) .
\end{aligned}
$$

Therefore, by (2.23) and (2.24) of Lemma 2.10, we infer

$$
\left|B_{2}(z, \bar{z})(\zeta)\right| \leq c d_{\bar{z}}(\bar{z}, z)^{\bar{\alpha}} d_{\bar{z}}(\bar{z}, \zeta)^{k-2}, \quad \forall z, \bar{z} \in E_{0}, \zeta \in \Omega_{M}(\bar{z}, z) .
$$

Finally, we observe that

$$
\begin{aligned}
B_{3}(z, \bar{z}) & =\left(L_{z} \varphi-L_{\bar{z}} \varphi\right)\left(u-P_{z}^{k-2} u\right)+L_{\bar{z}} \varphi\left(P_{\bar{z}}^{k-2} u-P_{z}^{k-2} u\right) \\
& =\left(P_{z}^{1} b-P_{\bar{z}}^{1} b\right) \partial_{y_{1}} \varphi\left(u-P_{z}^{k-2} u\right)+L_{\bar{z}} \varphi\left(P_{\bar{z}}^{k-2} u-P_{z}^{k-2} u\right) .
\end{aligned}
$$

Thus, applying again Lemma 2.10, we establish (4.19) and, consequently, (4.17).

We conclude the proof of (4.15) by showing that

$$
\left|D_{\sigma}(z) I_{1, z}-D_{\sigma}(\bar{z}) I_{1, \bar{z}}\right| \leq c d_{\bar{z}}(\bar{z}, z)^{\alpha}, \quad \forall \bar{z}, z \in E_{0},
$$

for some constant $c=c\left(E_{0}, k\right)$. We denote $\bar{\zeta}=(\bar{\xi}, \bar{\eta}, \bar{\tau})$ and by

$$
z \longmapsto R_{\zeta^{-1}}^{(\bar{z})}(z) \equiv z \circ \zeta^{-1}=\left(x-\xi, y-\eta+\mathcal{J}_{x} B(\bar{z})(\tau(x-\bar{x})-(\tau-\bar{t}) \xi), t-\tau\right),
$$


the right translation w.r.t. the law "o" in (2.11). By (4.6), for every $z \in E_{0}$ and multi-index $\sigma \in\{1, \ldots, N+1\}^{m}$ of weight $|\sigma|=k$, we have

$$
D_{\sigma}(z) I_{1, z}=\int \Gamma_{z}(\bar{\zeta}, 0) D_{\sigma}(z) U_{1, z}\left(R_{\bar{\zeta}^{-1}}^{(z)}(\cdot)\right) d \bar{\zeta}
$$

(by Lemma 3.2)

$$
=\sum_{|\mu| \leq k} \Lambda_{\mu, z}(z) \int \Gamma_{z}(\bar{\zeta}, 0) D_{\mu}^{z}(z) U_{1, z}\left(R_{\bar{\zeta}^{-1}}^{(z)}(\cdot)\right) d \bar{\zeta}
$$

(by Lemma 3.5)

$$
=\sum_{|\mu| \leq k} \sum_{\nu \in\{1, \ldots, 2 N+1\}^{m}} \Lambda_{\mu, z}(z) \int \Gamma_{z}(\bar{\zeta}, 0) P_{\nu, z}(\bar{\zeta}) D_{\nu}^{z}\left(z \circ \bar{\zeta}^{-1}\right) U_{1, z} d \bar{\zeta}
$$

(by the change of variables $\bar{\zeta}=\zeta^{-1} \circ z$ )

$$
=\int_{\Omega_{0}} \Gamma_{z}(z, \zeta) V_{z}(\zeta) d \zeta
$$

where

$$
V_{z}(\zeta)=\sum_{|\mu| \leq k} \sum_{\nu \in\{1, \ldots, 2 N+1\}^{m}} \Lambda_{\mu, z}(z) P_{\nu, z}\left(\zeta^{-1} \circ z\right) D_{\nu}^{z}(\zeta) U_{1, z} .
$$

We observe that

$$
\left|V_{z}(\zeta)-V_{\bar{z}}(\zeta)\right| \leq c d_{\bar{z}}(\bar{z}, z)^{\alpha}, \quad \forall z, \bar{z} \in E_{0}, \zeta \in \Omega_{0},
$$

for some constant $c$. Indeed, (4.23) follows from (3.8), the fact the function $z \mapsto P_{\nu, z}$ is of class $C_{B}^{\alpha}$ by Lemma 3.5 and the estimate

$$
\left|D_{\nu}^{z} P_{z}^{k} u(\zeta)-D_{\sigma}^{\bar{z}} P_{\bar{z}}^{k} u(\zeta)\right| \leq c d_{\bar{z}}(\bar{z}, z)^{\alpha} .
$$

At this point, the proof of (4.20) is analogous to the one of (4.17). Indeed it suffices to observe that

$$
D_{\sigma}(z) I_{1, z}-D_{\sigma}(\bar{z}) I_{1, \bar{z}}=A_{1}(z, \bar{z})+A_{2}(z, \bar{z})+A_{3}(z, \bar{z}),
$$

where

$$
\begin{aligned}
& A_{1}(z, \bar{z})=\int_{\Omega_{0} \backslash \Omega_{M}(\bar{z}, z)}\left(\Gamma_{z}(z, \zeta) V_{z}(\zeta)-\Gamma_{\bar{z}}(\bar{z}, \zeta) V_{\bar{z}}(\zeta)\right) d \zeta, \\
& A_{2}(z, \bar{z})=\int_{\Omega_{M}(\bar{z}, z)}\left(\Gamma_{z}(z, \zeta)-\Gamma_{\bar{z}}(\bar{z}, \zeta)\right) V_{\bar{z}}(\zeta) d \zeta, \\
& A_{3}(z, \bar{z})=\int_{\Omega_{M}(\bar{z}, z)} \Gamma_{z}(z, \zeta)\left(V_{z}(\zeta)-V_{\bar{z}}(\zeta)\right) d \zeta,
\end{aligned}
$$

and to conclude as before, by using Proposition 3.1. Thus, (4.15) is proved. We omit the proof of (4.16) since it is essentially analogous. 
The case $k=2$. Let $b, f \in C_{b}^{\alpha}(\Omega)$, with $0<\alpha<1$. Since, by assumption (1.10), $b \in C^{1}(\Omega)$, we have

$$
L_{B} u(z)-L_{\bar{z}} u(z)=\left(b-P_{\bar{z}}^{1} b\right) \partial_{y_{1}} u(z)=O\left(d_{\bar{z}}(\bar{z}, z)\right), \quad \text { as } z \longrightarrow \bar{z} .
$$

In this case, a much simpler choice of the frozen operators provides an approximation of $L_{b}$ of the same order. Indeed, for convenience, let us denote by $z=(x, y, t)$ a point of $\Omega$. For fixed $\bar{z} \in \Omega$, we define

$$
L^{(\bar{z})}=\Delta_{x}+\left(b(\bar{z})+x_{1}-\bar{x}_{1}\right) \partial_{y}-\partial_{t} .
$$

Then, $L^{(\bar{z})}$ is a Hörmander operator which, in the case $N=1$, up to a straightforward change of variables, coincides with the Kolmogorov operator in (1.5). Moreover, we have

$$
\begin{aligned}
L_{b} u(z)-L^{(\bar{z})} u(z) & =\left(b(z)-b(\bar{z})-\left(x_{1}-\bar{x}_{1}\right)\right) \partial_{y} u(z) \\
& =O\left(d^{(\bar{z})}(\bar{z}, z)\right), \quad \text { as } z \longrightarrow \bar{z},
\end{aligned}
$$

where $d^{(\bar{z})}$ denotes the control distance associated to $L^{(\bar{z})}$.

Given a cut-off function $\varphi$, we represent the solution $u$ in terms of the fundamental solution $\Gamma^{(\bar{z})}$ of $L^{(\bar{z})}$ :

$$
(u \varphi)(z)=\int_{\Omega} \Gamma^{(\bar{z})}(z, \zeta) L^{(\bar{z})}(u \varphi)(\zeta) d \zeta .
$$

Since $L^{(\bar{z})}(u \varphi) \in C_{0}(\Omega)$, it is standard to prove that $u \in C_{b}^{1, \bar{\alpha}}(\Omega)$, for every $\bar{\alpha} \in$ ]0,1[. Moreover, it is not difficult to adapt the previous arguments and to show that $u \in C_{b}^{2, \bar{\alpha}}(\Omega)$, for every $\left.\bar{\alpha} \in\right] 0, \alpha[$.

Remark 4.3. Theorem 1.3 holds true if we assume that $u$ is a locally Lipschitz continuous, strong solution to (1.1) instead of a classical solution. We recall that $u$ is a strong solution to (1.1) if it has weak derivatives and equation (1.1) is satisfied almost everywhere. In order to justify our claim, it suffices to remark that the proof of Theorem 1.3 is based only on the representation formula (4.2) and on the boundedness of the first-order derivatives of the solution.

\section{REFERENCES}

[1] Antonelli, F., Barucci, E., and Mancino, M. E. A Comparison result for FBSDE with Applications to Decisions Theory. Math. Methods Oper. Res. 2001, 54 (3), 407-423.

[2] Antonelli, F. and Pascucci, A. On the viscosity solutions of a stochastic differential utility problem. To appear in J. Differential Equations.

[3] Beals, R. $L^{p}$ and Hölder estimates for pseudodifferential operators: sufficient conditions. Ann. Inst. Fourier 1979, 29 (3), 239-260. MR 81c:47049

[4] Bramanti, M. and Brandolini, L. $L^{p}$ estimates for uniformly hypoelliptic operators with discontinuous coefficients on homogeneous groups. Trans. Amer. Math. Soc. 2000, 352 (2), $781-822$.

[5] Citti, G. $C^{\infty}$ regularity of solutions of a quasilinear equation related to the Levi operator. Ann. Scuola Norm. Sup. Pisa Cl. Sci., Serie IV 1996, 23, 483-529. MR 98b:35072

[6] Citti, G. and Montanari, A. $C^{\infty}$ regularity of solutions of an equation of Levi's type in $R^{2 n+1}$. Ann. Mat. Pura Appl.(4) 2001, 180 (1), 27-58. MR 2002f:35049

[7] Citti, G., Pascucci, A., and Polidoro, S. On the regularity of solutions to a nonlinear ultraparabolic equation arising in mathematical finance. Diff. Integral Equations 2001, 14 (6), 701-738. MR 2002f:35118

[8] Citti, G., Pascucci, A., and Polidoro, S. Regularity properties of viscosity solutions of a non-Hörmander degenerate equation. J. Math. Pures Appl. 2001, 80 (9), 901-918. 
[9] Escobedo, M., Vazquez, J. L., and Zuazua, E. Entropy solutions for diffusion-convection equations with partial diffusivity. Trans. Amer. Math. Soc. 1994, 343 (2), 829-842. MR 94h:35131

[10] Folland, G. B. Subelliptic estimates and function spaces on nilpotent Lie groups. Ark. Mat. 1975, 13, 161-207. MR 58:13215

[11] Hörmander, L. Hypoelliptic second order differential equations. Acta Math. 1967, 119, 147171. MR 36:5526

[12] Krylov, N. V. Hölder continuity and $L_{p}$ estimates for elliptic equations under general Hörmander's condition. Topological Methods Nonlinear Anal. 1997, 9 (2), 249-258. MR 99b:35077

[13] Lanconelli, E., Pascucci, A, and Polidoro, S. Linear and nonlinear ultraparabolic equations of Kolmogorov type arising in diffusion theory and in finance. To appear on "Nonlinear Problems in Mathematical Physics and Related Topics Vol. II In Honor of Professor O.A. Ladyzhenskaya". International Mathematical Series, Kluwer Ed.

[14] Lanconelli, E. and Polidoro, S. On a class of hypoelliptic evolution operators. Rend. Semin. Mat. Torino 1994, 52 (1), 29-63. MR 95h:35044

[15] Nagel A. and Stein, E. M. A new class of pseudodifferential operators. Proc. Nat. Acad. Sci. U.S.A. 1978, 75 (2), 582-585. MR 58:7222

[16] Nagel, A., Stein, E. M., and Wainger, S. Balls and metrics defined by vector fields I: Basic properties. Acta Math. 1985, 155, 103-147. MR 86k:46049

[17] Rothschild, L. P. and Stein, E. M. Hypoelliptic differential operators on nilpotent groups. Acta Math. 1977, 137, 247-320. MR 55:9171

[18] Shiryayev, A. N. (Ed.) Selected works of A. N. Kolmogorov. Vol. II. Probability theory and mathematical statistics. Kluwer Academic Publishers Group, Dordrecht, 1992, 597 pp. MR 92j:01071

[19] Xu, C. Regularity for quasilinear second-order subelliptic equations. Comm. Pure Appl. Math. 1992, 45, 77-96. MR 93b:35042

Dipartimento di Matematica, Università di Bologna, Piazza di Porta S. Donato 5, 40126 Bologna, ItALY

E-mail address: pascucci@dm.unibo.it 